\title{
False vacuum decay in gauge theory
}

\author{
Motoi Endo, ${ }^{a, b, c}$ Takeo Moroi, ${ }^{d, c}$ Mihoko M. Nojiri ${ }^{a, b, c}$ and Yutaro Shoji ${ }^{e}$ \\ ${ }^{a}$ KEK Theory Center, IPNS, KEK, \\ Tsukuba, Ibaraki 305-0801, Japan \\ ${ }^{b}$ The Graduate University of Advanced Studies (Sokendai), \\ Tsukuba, Ibaraki 305-0801, Japan \\ ${ }^{c}$ Kavli IPMU (WPI), University of Tokyo, \\ Kashiwa, Chiba 277-8583, Japan \\ ${ }^{d}$ Department of Physics, University of Tokyo, \\ Tokyo 113-0033, Japan \\ e Institute for Cosmic Ray Research, The University of Tokyo, \\ Kashiwa 277-8582, Japan \\ E-mail: motoi.endo@kek.jp, moroi@phys.s.u-tokyo.ac.jp, \\ nojiri@post.kek.jp, yshoji.hep@gmail.com
}

ABSTRACT: The decay rate of a false vacuum is studied in gauge theory, paying particular attention to its gauge invariance. Although the decay rate should not depend on the gauge parameter $\xi$ according to the Nielsen identity, the gauge invariance of the result of a perturbative calculation has not been clearly shown. We give a prescription to perform a one-loop calculation of the decay rate, with which a manifestly gauge-invariant expression of the decay rate is obtained. We also discuss the renormalization necessary to make the result finite, and show that the decay rate is independent of the gauge parameter even after the renormalization.

KEYwords: Gauge Symmetry, Solitons Monopoles and Instantons

ArXIV EPRINT: 1704.03492 


\section{Contents}

1 Introduction 1

2 Set up 3

2.1 Lagrangian, bounce 3

2.2 Fluctuation operators 5

$\begin{array}{lll}2.3 & \text { Prefactor } \mathcal{A} & 7\end{array}$

2.4 Functional determinant 8

3 Useful formulae $\quad 8$

$\begin{array}{lll}3.1 & \text { FP ghosts and transverse modes } & 9\end{array}$

$3.2 S, L$, and NG modes with $J \neq 0 \quad 9$

$\begin{array}{ll}3.3 & S \text { and NG modes with } J=0\end{array}$

4 Functional determinants: case with $v \neq 0 \quad 13$

$4.1 v \neq 0$ : contribution of $J \neq 0 \quad 13$

$\begin{array}{ll}4.2 v \neq 0 \text { : contribution of } J=0 & 15\end{array}$

$\begin{array}{lll}4.3 v \neq 0 \text { : final result } & 15\end{array}$

$5 \quad$ Functional determinants: case with $\boldsymbol{v}=\mathbf{0} \quad \mathbf{1 6}$

$\begin{array}{ll}5.1 v=0 \text { : contribution of } J \neq 0 & 16\end{array}$

$\begin{array}{ll}5.2 v=0 \text { : contribution of } J=0 & 19\end{array}$

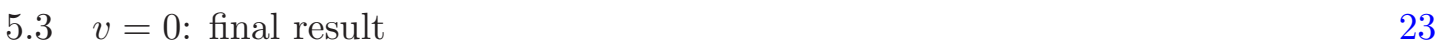

$\begin{array}{llr}6 & \text { Renormalization } & 23\end{array}$

$\begin{array}{lll}7 & \text { Conclusions and discussion } & 27\end{array}$

$\begin{array}{ll}\text { A Functional determinant } & 28\end{array}$

B Solutions of inhomogeneous differential equation 33

C Functional determinant with small perturbations $\quad 34$

\section{Introduction}

Calculation of the decay rate of a false vacuum (i.e., bubble nucleation rate) was formulated in [1-3] by introducing the so-called bounce, a saddle-point solution of the Euclidean classical equation of motion. The decay rate of the false vacuum per unit volume is expressed as

$$
\gamma=\mathcal{A} e^{-\mathcal{B}}
$$


Here, $\mathcal{B}$ is the bounce action, the Euclidean classical action of the bounce configuration. The prefactor $\mathcal{A}$ is obtained by integrating out field fluctuations around the bounce configuration as well as those around the false vacuum. It takes account of radiative corrections (i.e., loop corrections) to the effective action of the bounce.

When a scalar field responsible for the metastability of the false vacuum has gauge interactions, fluctuations of the gauge fields as well as those of the Faddeev-Popov (FP) ghosts contribute to the prefactor $\mathcal{A}$. Gauge dependence of $\mathcal{A}$ is the main subject of this paper. Gauge fixing is necessary for the calculation of the radiative corrections, with which a gauge parameter (which will be called $\xi$ in our analysis) is introduced. Then, some of the propagators of the fields acquire unphysical poles which depend on $\xi$; the $\xi$-dependence should vanish from physical quantities. According to the Nielsen identity, the effective action is gauge independent at its extrema $[4,5]$, although, in general, the effective action is gauge dependent. In perturbative calculations of the decay rate of the false vacuum, however, it has not been clarified how the gauge dependence vanishes and what the gauge invariant expression of the decay rate is.

The prefactor $\mathcal{A}$ consists of functional determinants of second-order differential operators (so-called fluctuation operators) governing mode functions of the field fluctuations. Such functional determinants are expressed by asymptotic values of solutions of the secondorder differential equations. Evaluation of the functional determinants has several complications in gauge theories. First, gauge and Nambu-Goldstone (NG) fields mix with each other when the gauge symmetry is spontaneously broken, which makes the behavior of the solutions complicated. Second, as we have already mentioned, the fluctuation operators contain the gauge parameter $\xi$ so that the $\xi$-independence of the decay rate is not manifest. These make it difficult not only to understand the gauge invariance but also to numerically calculate the decay rate. Indeed, for a stable numerical calculation, the 't Hooft-Feynman gauge with $\xi=1$ is usually adopted, with which the fluctuation operators become simple. However, with a calculation based on a particular choice of the gauge parameter, the gauge invariance of the result can not be discussed directly.

Recently, a gauge-invariant expression of the decay rate has been derived for a case where gauge symmetry is spontaneously broken in the false vacuum [6]. In [6], a gauge fixing function which reduces to the $R_{\xi}$ gauge around the false vacuum has been adopted. (We call such a gauge as an $R_{\xi}$-like gauge.) However the procedure proposed in [6] cannot be applied if gauge symmetry is preserved in the false vacuum. This is because, in such a case, there shows up a class of bounce configurations related by the internal symmetry, all of which contribute to the false vacuum decay. With the $R_{\xi}$-like gauge fixing, the fluctuation operators are dependent on the bounce configuration, which makes it difficult to take account of effects of all the possible bounce configurations.

In this paper, we study the decay of the false vacuum in 4-dimensional (4D) gauge theory, paying particular attention to the gauge invariance of the decay rate. ${ }^{1}$ We improve the analysis of [6] and present a prescription giving rise to a gauge-invariant expression of the decay rate, which is applicable to the symmetry-preserving false vacuum. We use the

\footnotetext{
${ }^{1}$ For the study of the thermal transition rate of sphaleron, see [7].
} 
following gauge fixing function, $\mathcal{F}=\partial_{\mu} A_{\mu}$ (with $A_{\mu}$ being the gauge field), with which we show that the difficulty mentioned above can be avoided. We resolve the complication due to the mixing between the gauge and NG modes, and systematically integrate out the fluctuations of the gauge field, NG mode, and the FP ghosts to calculate the prefactor $\mathcal{A}$. We give a manifestly gauge invariant expression of the decay rate of the false vacuum. Our results are useful not only for understanding the gauge invariance of the decay rate but also for simplifying the numerical calculation of the decay rate.

The organization of this paper is as follows. In section 2, we describe the model we consider. Important formulae for our analysis are discussed in section 3. Calculations of the functional determinants for the cases with and without the spontaneous symmetry breaking of the gauge symmetry at the false vacuum are given in sections 4 and 5 , respectively. The issues related to the renormalization are studied in 6 . The final expression of the decay rate can be derived from eqs. (6.22)-(6.26); readers who are interested only in the result can skip to these equations. Section 7 is devoted for conclusions and discussion.

\section{Set up}

\subsection{Lagrangian, bounce}

We consider a model with U(1) gauge symmetry; application of our argument to the case with non-abelian gauge groups is straightforward. For simplicity, we concentrate on the case where there exists only one charged scalar field $\Phi$ which acquires a vacuum expectation value (VEV). The Euclidean Lagrangian is given in the following form:

$$
\mathcal{L}=\frac{1}{4} F_{\mu \nu} F_{\mu \nu}+\left[\left(\partial_{\mu}+i g A_{\mu}\right) \Phi^{\dagger}\right]\left[\left(\partial_{\mu}-i g A_{\mu}\right) \Phi\right]+V+\mathcal{L}_{\text {G.F. }}+\mathcal{L}_{\text {ghost }},
$$

where $F_{\mu \nu}=\partial_{\mu} A_{\nu}-\partial_{\nu} A_{\mu}$, and $V$ is the scalar potential. In addition, $\mathcal{L}_{\text {G.F. }}$ and $\mathcal{L}_{\text {ghost }}$ are the gauge fixing terms and the Lagrangian of the FP ghosts, respectively. The scalar potential $V$ has true and false vacua. We denote the scalar amplitude at the false vacuum to be $v / \sqrt{2}$, and choose the field configuration of the false vacuum as

$$
\left(A_{\mu}, \Phi\right)_{\text {false vacuum }}=(0, v / \sqrt{2}) .
$$

In the path integral formulation, the decay of the false vacuum is dominated by a classical path, i.e., an $O(4)$ symmetric solution of the $4 \mathrm{D}$ Euclidean classical equation of motion with appropriate boundary conditions (the so-called bounce) [1-3]. The bounce solution settles to the false vacuum at the infinity of the Euclidean space:

$$
\lim _{r \rightarrow \infty}\left(A_{\mu}, \Phi\right)_{\text {bounce }}=(0, v / \sqrt{2}),
$$

where $r \equiv \sqrt{x_{\mu} x_{\mu}}$ is the $4 \mathrm{D}$ radius in the Euclidean space. The bounce is characterized by the function $\bar{\phi}(r)$ which obeys

$$
\left[\partial_{r}^{2} \Phi+\frac{3}{r} \partial_{r} \Phi-V_{\Phi}\right]_{\Phi \rightarrow \bar{\phi} / \sqrt{2}}=0
$$


where $V_{\Phi} \equiv \partial V / \partial \Phi$. It also satisfies

$$
\begin{aligned}
\partial_{r} \bar{\phi}(r=0) & =0, \\
\bar{\phi}(r=\infty) & =v,
\end{aligned}
$$

where the center of the bounce is set to be $r=0$. We assume that $\bar{\phi}$ is a real function of $r$. The bounce configuration is given by $\left(A_{\mu}, \Phi\right)=(0, \bar{\phi} / \sqrt{2})$ when $v \neq 0$, while that for the case of $v=0$ is not unique as we will explain later.

It is important to understand asymptotic behavior of the function $\bar{\phi}$. Let us assume that the leading term of the scalar potential around the false vacuum is quadratic. Then, because $\bar{\phi}$ settles to the false-vacuum amplitude at $r \rightarrow \infty$, the asymptotic behavior of $\bar{\phi}$ can be understood by using the following equation:

$$
\partial_{r}^{2} \bar{\phi}+\frac{3}{r} \partial_{r} \bar{\phi}-m_{\phi}^{2}(\bar{\phi}-v) \simeq 0,
$$

where $m_{\phi}^{2}$ is the curvature of the potential around the false vacuum. We parameterize the asymptotic behavior of $\bar{\phi}$ as

$$
\bar{\phi}(r \rightarrow \infty) \simeq v+\kappa \frac{e^{-m_{\phi} r}}{r^{3 / 2}}
$$

where $\kappa$ is a constant.

In some of the previous studies [8,9], a gauge fixing function which reduces to that of the $R_{\xi}$ gauge in the false vacuum has been adopted: $\mathcal{F}^{\left(R_{\xi}\right)}=\partial_{\mu} A_{\mu}-2 \xi g(\operatorname{Re} \Phi)(\operatorname{Im} \Phi)$. However, such a gauge fixing function causes a problem when $v=0$ [6]. In such a case, the symmetry is restored in the false vacuum and there appears a class of independent bounce configurations related by the internal $\mathrm{U}(1)$ symmetry. The configuration is given by $\left(A_{\mu}, \Phi\right)=\left(\partial_{\mu} \Theta / g, \bar{\phi} e^{i \Theta} / \sqrt{2}\right)$, where the function $\Theta$ obeys

$$
\partial_{r}^{2} \Theta+\frac{3}{r} \partial_{r} \Theta-\frac{1}{2} \xi g^{2} \bar{\phi}^{2} \sin 2 \Theta=0 .
$$

The above configuration satisfies the classical equation of motion as well as the boundary condition given in eq. (2.3). The function $\Theta$ is required to be finite, and is determined by its value at $r=0$; an independent set of the bounce configurations is obtained with $0 \leq \Theta(0)<2 \pi$. When $v=0$, we should take account of all the bounce configurations labeled by $\Theta(0)$ for the calculation of the decay rate of the false vacuum. However, it is highly non-trivial because the fluctuation operators around the bounce depend on $\Theta(0)$, and also because we have to understand the measure for the integration over $\Theta(0)$.

Such complications can be avoided with a gauge fixing function which does not contain the scalar field [10]. In our analysis, we adopt the following gauge fixing function:

$$
\mathcal{F}=\partial_{\mu} A_{\mu},
$$

with which the gauge fixing term is given by

$$
\mathcal{L}_{\text {G.F. }}=\frac{1}{2 \xi} \mathcal{F}^{2} .
$$


In addition, the FP ghosts become free fields:

$$
\mathcal{L}_{\text {ghost }}=-\bar{c} \partial_{\mu} \partial_{\mu} c
$$

With the gauge fixing function given in eq. (2.10), we find a class of solution of the classical equation of motion, which is given by

$$
\left(A_{\mu}, \Phi\right)_{\text {classical solution }}=\left(0, \bar{\phi}(r) e^{i \vartheta} / \sqrt{2}\right)
$$

with $\vartheta$ being a constant, parameterizing the configurations of the classical solutions. When $v \neq 0$, only one configuration with $\vartheta=0$ satisfies the boundary condition given in eq. (2.3). Then, we can easily integrate out the fluctuations around the bounce, as we will discuss in section 4 . On the contrary, when $v=0$, all the classical solutions parameterized by $\vartheta$ contribute to the false-vacuum decay because all the bounce parameterized by $\vartheta$ has the same asymptotic value at $r \rightarrow \infty$, and contributes to the vacuum decay. This issue will be discussed in section 5 .

\section{$2.2 \quad$ Fluctuation operators}

For the evaluation of the decay rate of the false vacuum, it is necessary to integrate out the fluctuations around the bounce. Such an integration can be performed by calculating the functional determinants of the second order differential operators (i.e., fluctuation operators). It is convenient to decompose the gauge field and $\Phi$ as

$$
A_{\mu}=a_{\mu}, \quad \Phi=\frac{1}{\sqrt{2}} e^{i \vartheta}(\bar{\phi}+h+i \varphi)
$$

with $h$ and $\varphi$ being real modes. Hereafter, we call $h$ and $\varphi$ as "Higgs" and "NG" modes, respectively.

With the gauge fixing function given in eq. (2.10), the fluctuation operator of the gauge and NG modes around the bounce configuration is

$$
\mathcal{M}^{\left(A_{\mu}, \varphi\right)} \equiv\left(\begin{array}{cc}
-\partial^{2} \delta_{\mu \nu}+\left(1-\frac{1}{\xi}\right) \partial_{\mu} \partial_{\nu}+g^{2} \bar{\phi}^{2} & g\left(\partial_{\nu} \bar{\phi}\right)-g \bar{\phi} \partial_{\nu} \\
2 g\left(\partial_{\mu} \bar{\phi}\right)+g \bar{\phi} \partial_{\mu} & -\partial^{2}+\frac{\left(\partial^{2} \bar{\phi}\right)}{\bar{\phi}}
\end{array}\right) .
$$

Here, the derivatives act on everything to the right unless brackets exist; for example, with the expression $\left(\partial_{\mu} \bar{\phi}\right)$, the derivative acts only on $\bar{\phi}$. The calculation of the functional determinant of $\mathcal{M}^{\left(A_{\mu}, \varphi\right)}$ is the main subject of this paper.

Because the bounce configuration is $O(4)$ symmetric [11, 12], the fluctuations can be expanded by using hyperspherical functions on $S^{3}$. The hyperspherical functions are labeled by the quantum numbers of the rotational group of the $4 \mathrm{D}$ Euclidean space, i.e., $\mathrm{SU}(2)_{A} \times$ $\mathrm{SU}(2)_{B}$ (the so-called $A$ - and $B$-spins). Namely, we denote $\mathcal{Y}_{J, m_{A}, m_{B}} \equiv\left\langle\hat{\mathbf{r}} \mid J, m_{A}, m_{B}\right\rangle$; $\hat{\mathbf{r}}$ is the coordinate on $S^{3}$, the eigenvalue of $S_{A}^{2}$ and $S_{B}^{2}$ is $J(J+1)$, and that of $S_{A, 3}$ $\left(S_{B, 3}\right)$ is $m_{A}\left(m_{B}\right)$, where generators of $\mathrm{SU}(2)_{A}$ and $\mathrm{SU}(2)_{B}$ are denoted as $S_{A}$ and $S_{B}$, 
respectively. With introducing radial mode functions denoted as $\alpha_{S}, \alpha_{L}, \alpha_{T 1}$, and $\alpha_{T 2}, a_{\mu}$ can be expanded as

$$
\begin{aligned}
a_{\mu}(x) \ni & \alpha_{S}(r) \frac{x_{\mu}}{r} \mathcal{Y}_{J, m_{A}, m_{B}}+\alpha_{L}(r) \frac{r}{L} \partial_{\mu} \mathcal{Y}_{J, m_{A}, m_{B}} \\
& +\alpha_{T 1}(r) i \epsilon_{\mu \nu \rho \sigma} V_{\nu}^{(1)} L_{\rho \sigma} \mathcal{Y}_{J, m_{A}, m_{B}}+\alpha_{T 2}(r) i \epsilon_{\mu \nu \rho \sigma} V_{\nu}^{(2)} L_{\rho \sigma} \mathcal{Y}_{J, m_{A}, m_{B}}
\end{aligned}
$$

where $V_{\nu}^{(1)}$ and $V_{\nu}^{(2)}$ are (arbitrary) two independent vectors, $L_{\rho \sigma} \equiv \frac{i}{\sqrt{2}}\left(x_{\rho} \partial_{\sigma}-x_{\sigma} \partial_{\rho}\right)$, and

$$
L \equiv \sqrt{4 J(J+1)} .
$$

(Notice that $L_{\mu \nu} L_{\mu \nu} \mathcal{Y}_{J, m_{A}, m_{B}}=L^{2} \mathcal{Y}_{J, m_{A}, m_{B}}$.) Here, we omit subscripts $J, m_{A}$, and $m_{B}$ from the mode functions for notational simplicity, and summations over $J, m_{A}$, and $m_{B}$ are implicit. There is no $L$ - or $T$-mode for $J=0$. The scalar bosons can be expanded as

$$
\begin{aligned}
& h(x) \ni \alpha_{h}(r) \mathcal{Y}_{J, m_{A}, m_{B}}, \\
& \varphi(x) \ni \alpha_{\varphi}(r) \mathcal{Y}_{J, m_{A}, m_{B}} .
\end{aligned}
$$

The behavior of the radial mode functions are governed by the fluctuation operators. In the following, we show explicit expressions of the fluctuation operators for each angular momentum.

The fluctuation operator for $\left(\alpha_{S}, \alpha_{L}, \alpha_{\varphi}\right)$ and that for $\left(\alpha_{T 1}, \alpha_{T 2}\right)$ are decoupled from each other. For $J>0$, the fluctuation operator for $\left(\alpha_{S}, \alpha_{L}, \alpha_{\varphi}\right)$ is given by

$$
\begin{aligned}
\mathcal{M}_{J}^{(S, L, \varphi)} \equiv & \left(\begin{array}{ccc}
-\Delta_{J}+\frac{3}{r^{2}}+g^{2} \bar{\phi}^{2} & -\frac{2 L}{r^{2}} & g \bar{\phi}^{\prime}-g \bar{\phi} \partial_{r} \\
-\frac{2 L}{r^{2}} & -\Delta_{J}-\frac{1}{r^{2}}+g^{2} \bar{\phi}^{2} & -\frac{L}{r} g \bar{\phi} \\
2 g \bar{\phi}^{\prime}+g \bar{\phi} \partial_{r}+\frac{3}{r} g \bar{\phi} & -\frac{L}{r} g \bar{\phi} & -\Delta_{J}+\frac{\left(\Delta_{0} \bar{\phi}\right)}{\bar{\phi}}
\end{array}\right) \\
& +\left(1-\frac{1}{\xi}\right)\left(\begin{array}{ccc}
\partial_{r}^{2}+\frac{3}{r} \partial_{r}-\frac{3}{r^{2}}-L\left(\frac{1}{r} \partial_{r}-\frac{1}{r^{2}}\right) & 0 \\
L\left(\frac{1}{r} \partial_{r}+\frac{3}{r^{2}}\right) & -\frac{L^{2}}{r^{2}} & 0 \\
0 & 0 & 0
\end{array}\right),
\end{aligned}
$$

where $\bar{\phi}^{\prime} \equiv \partial_{r} \bar{\phi}$, and

$$
\Delta_{J} \equiv \partial_{r}^{2}+\frac{3}{r} \partial_{r}-\frac{L^{2}}{r^{2}}
$$

For $J=0, \alpha_{L}$-mode does not exist, and the fluctuation operator is in the form of $2 \times 2$ differential operator; $\mathcal{M}_{J=0}^{(S, \varphi)}$ is obtained from eq. (2.20) by eliminating the second row and the second column:

$$
\mathcal{M}_{J=0}^{(S, \varphi)} \equiv\left(\begin{array}{cc}
\frac{1}{\bar{\xi}}\left(-\Delta_{0}+\frac{3}{r^{2}}+\xi g^{2} \bar{\phi}^{2}\right) & g \bar{\phi}^{\prime}-g \bar{\phi} \partial_{r} \\
2 g \bar{\phi}^{\prime}+g \bar{\phi} \partial_{r}+\frac{3}{r} g \bar{\phi} & -\Delta_{0}+\frac{\left(\Delta_{0} \bar{\phi}\right)}{\bar{\phi}}
\end{array}\right) .
$$


In addition, the fluctuation operator of the transverse modes is given by

$$
\mathcal{M}_{J}^{(T)}=-\Delta_{J}+g^{2} \bar{\phi}^{2}
$$

while that of the FP ghosts is

$$
\mathcal{M}_{J}^{(\bar{c}, c)}=-\Delta_{J}
$$

The radial mode functions can be expanded by using the eigenfunctions of these fluctuation operators.

We also need fluctuation operators around the false vacuum, which are denoted as $\widehat{\mathcal{M}}^{\left(A_{\mu}, \varphi\right)}, \widehat{\mathcal{M}}_{J}^{(S, L, \varphi)}, \widehat{\mathcal{M}}_{J}^{(T)}$, and so on. (In this paper, the "hat" is used for objects around the false vacuum.) They can be obtained from the corresponding fluctuation operators around the bounce by replacing $\bar{\phi} \rightarrow v$, and $\bar{\phi}^{\prime} \rightarrow 0$. For the case of $v=0,\left(\Delta_{0} \bar{\phi}\right) / \bar{\phi}$ should be replaced by $m_{\phi}^{2}$.

Finally, let us comment on the contribution of the Higgs mode. In this paper, we concentrate on the case where there exists only one charged scalar field which has nonvanishing amplitude. However, assuming renormalizability, extra neutral scalars are necessary to make the scalar potential to have both false and true vacua; we implicitly assume that this is the case. The neutral scalar fields may mix with the charged scalar when the $\mathrm{U}(1)$ symmetry is broken. Thus, the fluctuation operators of the CP-even scalars (which includes the Higgs mode) are highly model dependent. ${ }^{2}$ However, the fluctuation operators of the CP-even scalars do not depend on $\xi$, and hence have nothing to do with the gauge dependence of the decay rate of the false vacuum, which is of our primary concern. Therefore, we do not discuss the effects of the Higgs mode.

\section{$2.3 \quad$ Prefactor $\mathcal{A}$}

The prefactor $\mathcal{A}$ in eq. (1.1) is given by [2]

$$
\mathcal{A}=\frac{\mathcal{B}^{2}}{4 \pi^{2}} \mathcal{A}^{\prime(h)} \mathcal{A}^{\left(A_{\mu}, \varphi\right)} \mathcal{A}^{(\bar{c}, c)} \mathcal{A}^{(\text {extra })} e^{-\mathcal{S}^{(\text {c.t. })},}
$$

where $\mathcal{A}^{\prime(h)}, \mathcal{A}^{\left(A_{\mu}, \varphi\right)}$, and $\mathcal{A}^{(\bar{c}, c)}$ are contributions of the Higgs mode, $\left(A_{\mu}, \varphi\right)$, and the FP ghosts, respectively. The "prime" on $\mathcal{A}^{\prime(h)}$ indicates that the effect of the zero modes in association with the translational invariance is omitted [2]. If there exist extra fields other than those mentioned above, their contribution is denoted as $\mathcal{A}^{(\text {extra) }}$; hereafter, we do not consider $\mathcal{A}^{\text {(extra) }}$. In addition, $\mathcal{S}^{\text {(c.t.) }}$ is the counter term to subtract the divergences.

At the one-loop level, each contribution is obtained by evaluating the functional determinants of the fluctuation operators. In particular, formally, $\mathcal{A}^{\left(A_{\mu}, \varphi\right)}$ is given in the following form

$$
\mathcal{A}^{\left(A_{\mu}, \varphi\right)}=\left[\frac{\operatorname{Det} \mathcal{M}^{\left(A_{\mu}, \varphi\right)}}{\operatorname{Det} \widehat{\mathcal{M}}^{\left(A_{\mu}, \varphi\right)}}\right]^{-1 / 2} .
$$

\footnotetext{
${ }^{2}$ In our study, we assume no significant $\mathrm{CP}$ violation in the scalar sector, i.e., no mixing between CP-even and CP-odd scalars.
} 
It can be further decomposed into the contributions of $\left(\alpha_{S}, \alpha_{L}, \varphi\right)$ and $\left(\alpha_{T 1}, \alpha_{T 2}\right)$, which are denoted as $\mathcal{A}^{(S, L, \varphi)}$ and $\mathcal{A}^{(T)}$, respectively, as

$$
\mathcal{A}^{\left(A_{\mu}, \varphi\right)}=\mathcal{A}^{(S, L, \varphi)} \mathcal{A}^{(T)},
$$

where

$$
\begin{aligned}
\mathcal{A}^{(S, L, \varphi)} & =\left[\frac{\operatorname{Det} \mathcal{M}_{0}^{(S, \varphi)}}{\operatorname{Det} \widehat{\mathcal{M}}_{0}^{(S, \varphi)}}\right]^{-1 / 2} \prod_{J=1 / 2}^{\infty}\left[\frac{\operatorname{Det} \mathcal{M}_{J}^{(S, L, \varphi)}}{\operatorname{Det} \widehat{\mathcal{M}}_{J}^{(S, L, \varphi)}}\right]^{-(2 J+1)^{2} / 2}, \\
\mathcal{A}^{(T)} & =\prod_{J=1 / 2}^{\infty}\left[\frac{\operatorname{Det} \mathcal{M}_{J}^{(T)}}{\operatorname{Det} \widehat{\mathcal{M}}_{J}^{(T)}}\right]^{-(2 J+1)^{2}}
\end{aligned}
$$

Furthermore, the ghost contribution is given by

$$
\mathcal{A}^{(\bar{c}, c)}=\prod_{J=0}^{\infty}\left[\frac{\operatorname{Det} \mathcal{M}_{J}^{(\bar{c}, c)}}{\operatorname{Det} \widehat{\mathcal{M}}_{J}^{(\bar{c}, c)}}\right]^{(2 J+1)^{2}} .
$$

\subsection{Functional determinant}

In order to evaluate the functional determinants, we use the method given in [3, 13-15]. For fluctuation operators $\mathcal{M}_{J}^{(X)}$ and $\widehat{\mathcal{M}}_{J}^{(X)}$, which are $N \times N$ differential operators in general, we first consider $N$ independent functions $\psi_{I}^{(X)}(r)$ and $\widehat{\psi}_{I}^{(X)}(r)$ (with $I=1-N$ ), which obey

$$
\begin{aligned}
& \mathcal{M}_{J}^{(X)} \psi_{I}^{(X)}=0, \\
& \widehat{\mathcal{M}}_{J}^{(X)} \widehat{\psi}_{I}^{(X)}=0 .
\end{aligned}
$$

In addition, $\psi_{I}^{(X)}$ and $\widehat{\psi}_{I}^{(X)}$ are regular at $r \rightarrow 0$. With these functions, we introduce the following quantities:

$$
\begin{aligned}
& \mathcal{D}_{J}^{(X)}(r) \equiv \operatorname{det}\left(\psi_{1}^{(X)}(r) \cdots \psi_{N}^{(X)}(r)\right), \\
& \widehat{\mathcal{D}}_{J}^{(X)}(r) \equiv \operatorname{det}\left(\widehat{\psi}_{1}^{(X)}(r) \cdots \widehat{\psi}_{N}^{(X)}(r)\right) .
\end{aligned}
$$

When $\psi_{I}^{(X)}(r)$ and $\widehat{\psi}_{I}^{(X)}(r)$ have the same boundary condition at $r \rightarrow 0$, the quantity $\operatorname{Det} \mathcal{M}_{J}^{(X)} / \operatorname{Det} \widehat{\mathcal{M}}_{J}^{(X)}$ is given by the ratio of $\mathcal{D}_{J}^{(X)}(r \rightarrow \infty)$ and $\widehat{\mathcal{D}}_{J}^{(X)}(r \rightarrow \infty)$. For a general boundary condition at $r=0$, the ratio of the functional determinants is given by

$$
\frac{\operatorname{Det} \mathcal{M}_{J}^{(X)}}{\operatorname{Det} \widehat{\mathcal{M}}_{J}^{(X)}}=\left[\frac{\mathcal{D}_{J}^{(X)}(r \rightarrow 0)}{\widehat{\mathcal{D}}_{J}^{(X)}(r \rightarrow 0)}\right]^{-1} \frac{\mathcal{D}_{J}^{(X)}\left(r_{\infty}\right)}{\widehat{\mathcal{D}}_{J}^{(X)}\left(r_{\infty}\right)},
$$

where $r_{\infty}$ is the abbreviation of $r \rightarrow \infty$. This relation is derived in appendix A.

\section{Useful formulae}

In this section, we summarize properties of the functions used in our calculations of the functional determinants in later sections. The formulae given in this section are applicable both for $v \neq 0$ and $v=0$. 


\subsection{FP ghosts and transverse modes}

The fluctuation operator for the FP ghosts is given in eq. (2.24). We define the function $f_{J}^{(\mathrm{FP})}$ which obeys

$$
\Delta_{J} f_{J}^{(\mathrm{FP})}=0
$$

and $f_{J}^{(\mathrm{FP})}(0)$ is required to be finite; we normalize $f_{J}^{(\mathrm{FP})}$ as

$$
f_{J}^{(\mathrm{FP})}(r)=r^{2 J}
$$

(For $J=0, f_{0}^{(\mathrm{FP})}(r)=1$.) The differential equation $\widehat{\mathcal{M}}_{J}^{(\bar{c}, c)} \widehat{f}_{J}^{(\mathrm{FP})}=0$ has the same solution:

$$
\widehat{f}_{J}^{(\mathrm{FP})}(r)=f_{J}^{(\mathrm{FP})}(r) \text {. }
$$

Using eq. (2.35),

$$
\frac{\operatorname{Det} \mathcal{M}_{J}^{(\bar{c}, c)}}{\operatorname{Det} \widehat{\mathcal{M}}_{J}^{(\bar{c}, c)}}=1 .
$$

For the functional determinants for the transverse modes, we define the functions $f_{J}^{(T)}$ and $\widehat{f}_{J}^{(T)}$, obeying

$$
\begin{aligned}
& \left(\Delta_{J}-g^{2} \bar{\phi}^{2}\right) f_{J}^{(T)}=0, \\
& \left(\Delta_{J}-g^{2} v^{2}\right) \widehat{f}_{J}^{(T)}=0,
\end{aligned}
$$

with

$$
f_{J}^{(T)}(r \rightarrow 0) \simeq \widehat{f}_{J}^{(T)}(r \rightarrow 0) \simeq r^{2 J} .
$$

Then,

$$
\frac{\operatorname{Det} \mathcal{M}_{J}^{(T)}}{\operatorname{Det} \widehat{\mathcal{M}}_{J}^{(T)}}=\frac{f_{J}^{(T)}\left(r_{\infty}\right)}{\widehat{f}_{J}^{(T)}\left(r_{\infty}\right)}
$$

\section{2 $S, L$, and NG modes with $J \neq 0$}

In this subsection, we consider the functional determinants of the fluctuation operators for the $S, L$, and NG modes with $J \neq 0$. For this purpose, we should consider the equations

$$
\mathcal{M}_{J}^{(S, L, \varphi)} \Psi=0,
$$

and

$$
\widehat{\mathcal{M}}_{J}^{(S, L, \varphi)} \widehat{\Psi}=0 .
$$


First, we consider eq. (3.9). It is convenient to use the fact that the solutions of eq. (3.9) can be expressed by using three functions which we call $\chi, \eta$, and $\zeta$. Let us define

$$
\Psi \equiv\left(\begin{array}{c}
\Psi^{(\text {top })} \\
\Psi^{(\mathrm{mid})} \\
\Psi^{(\mathrm{bot})}
\end{array}\right) \equiv\left(\begin{array}{c}
\partial_{r} \chi \\
\frac{L}{r} \chi \\
g \bar{\phi} \chi
\end{array}\right)+\left(\begin{array}{c}
\frac{1}{r g^{2} \bar{\phi}^{2}} \eta \\
\frac{1}{L r^{2} g^{2} \bar{\phi}^{2}} \partial_{r}\left(r^{2} \eta\right) \\
0
\end{array}\right)+\left(\begin{array}{c}
-2 \frac{\bar{\phi}^{\prime}}{g^{2} \bar{\phi}^{3}} \zeta \\
0 \\
\frac{1}{g \bar{\phi}} \zeta
\end{array}\right)
$$

where the functions $\chi, \eta$, and $\zeta$ obey the following equations:

$$
\begin{aligned}
\Delta_{J} \chi & =\frac{2 \bar{\phi}^{\prime}}{r g^{2} \bar{\phi}^{3}} \eta+\frac{2}{r^{3}} \partial_{r}\left(\frac{r^{3} \bar{\phi}^{\prime}}{g^{2} \bar{\phi}^{3}} \zeta\right)-\xi \zeta, \\
\left(\Delta_{J}-g^{2} \bar{\phi}^{2}\right) \eta-\frac{2 \bar{\phi}^{\prime}}{r^{2} \bar{\phi}} \partial_{r}\left(r^{2} \eta\right) & =-\frac{2 L^{2} \bar{\phi}^{\prime}}{r \bar{\phi}} \zeta, \\
\Delta_{J} \zeta & =0 .
\end{aligned}
$$

Then, using the equation for the bounce solution given in eq. (2.4), we can show that $\Psi$ satisfies eq. (3.9). Thus, the function $\Psi$ given in eq. (3.11) has the right property to calculate the functional determinant of $\mathcal{M}_{J}^{(S, L, \varphi)}$, assuming that $\Psi(r=0)$ is finite. In addition, the following equations hold for the top and middle components of $\Psi$, which are also useful for the following argument:

$$
\begin{aligned}
\partial_{r} \Psi^{(\mathrm{top})} & =-\frac{3}{r} \Psi^{(\mathrm{top})}+\frac{L}{r} \Psi^{(\mathrm{mid})}-\xi \zeta, \\
\partial_{r} \Psi^{(\mathrm{mid})} & =\frac{L}{r} \Psi^{(\mathrm{top})}-\frac{1}{r} \Psi^{(\mathrm{mid})}+\frac{1}{L} \eta .
\end{aligned}
$$

Notice that eq. (3.15) can be translated to $\alpha_{\mathcal{F}}+\xi \zeta=0$, where $\alpha_{\mathcal{F}}$ is the radial mode function of the gauge fixing function, i.e., $\mathcal{F}(x) \ni \alpha_{\mathcal{F}}(r) \mathcal{Y}_{J, m_{A}, m_{B}}$.

If three independent solutions of eq. (3.9) are given (which we call $\Psi_{I}$ with $I=1$, 2 , and 3), the functional determinant of $\mathcal{M}_{J}^{(S, L, \varphi)}$ can be related to the function $\mathcal{D}_{J}^{(S, L, \varphi)}$ defined as

$$
\mathcal{D}_{J}^{(S, L, \varphi)}(r) \equiv \operatorname{det}\left(\Psi_{1}(r) \Psi_{2}(r) \Psi_{3}(r)\right) .
$$

We consider the following three independent solutions, which are composed of the functions $\chi_{I}, \eta_{I}$, and $\zeta_{I}$.

1. We take $\eta_{1}=\zeta_{1}=0$; this condition is consistent with eqs. (3.13) and (3.14). Then, eq. (3.12) can be easily solved to obtain $\chi_{1}$ by requiring its regularity at the origin; we normalize $\chi_{1}$ as

$$
\chi_{1}(r)=r^{2 J}
$$

which gives

$$
\Psi_{1}(r)=\left(\begin{array}{c}
2 J r^{2 J-1} \\
L r^{2 J-1} \\
g \bar{\phi} r^{2 J}
\end{array}\right)
$$


2. We take $\zeta_{2}=0$ (which is consistent with (3.14)), while $\chi_{2}$ and $\eta_{2}$ are non-vanishing. For the boundary conditions at $r \rightarrow 0$, we take

$$
\begin{aligned}
& \chi_{2}(r \rightarrow 0) \simeq-\frac{1}{2 J g^{2} \bar{\phi}_{C}^{2}} r^{2 J}, \\
& \eta_{2}(r \rightarrow 0) \simeq r^{2 J},
\end{aligned}
$$

where $\bar{\phi}_{C}$ is the scalar amplitude at the center of the bounce:

$$
\bar{\phi}_{C} \equiv \bar{\phi}(r=0) .
$$

We find

$$
\Psi_{2}(r \rightarrow 0) \simeq\left(\begin{array}{c}
\frac{1}{8(J+1)} r^{2 J+1} \\
\frac{J(J+2)}{L^{3}} r^{2 J+1} \\
-\frac{1}{2 J g \bar{\phi}_{C}} r^{2 J}
\end{array}\right)
$$

3. For $\Psi_{3}$, we take

$$
\zeta_{3}(r)=r^{2 J},
$$

and $\chi_{3}(r \rightarrow 0) \sim \eta_{3}(r \rightarrow 0) \sim O\left(r^{2 J+2}\right)$. Then, we find

$$
\Psi_{3}(r \rightarrow 0) \simeq\left(\begin{array}{c}
-\frac{1}{4} \xi r^{2 J+1} \\
-\frac{J}{2 L} \xi r^{2 J+1} \\
\frac{1}{g \bar{\phi}_{C}} r^{2 J}
\end{array}\right) .
$$

Combining eqs. (3.19), (3.23), and (3.25), we obtain

$$
\mathcal{D}_{J}^{(S, L, \varphi)}(r \rightarrow 0) \simeq \frac{2 J[(J+1) \xi+J]}{L^{3} g \bar{\phi}_{C}} r^{6 J} .
$$

In order to express the homogeneous solution of eq. (3.13) with $\zeta=0$, we introduce the function $f_{J}^{(\eta)}$, which obeys

$$
\left(\Delta_{J}-g^{2} \bar{\phi}^{2}\right) f_{J}^{(\eta)}-\frac{2 \bar{\phi}^{\prime}}{r^{2} \bar{\phi}} \partial_{r}\left(r^{2} f_{J}^{(\eta)}\right)=0
$$

whose boundary condition is taken to be

$$
f_{J}^{(\eta)}(r \rightarrow 0) \simeq r^{2 J}
$$

$\left(\right.$ For $J=0, f_{0}^{(\eta)}(r \rightarrow 0) \simeq 1$.) At $r \rightarrow \infty, f_{J}^{(\eta)}$ behaves as

$$
f_{J}^{(\eta)}(r \rightarrow \infty) \simeq c_{\eta} \frac{e^{g v r}}{r^{3 / 2}}\left[1+O\left(r^{-1}\right)\right]
$$


where $c_{\eta}$ is a constant. We emphasize here that the function $f_{J}^{(\eta)}$ is independent of $\xi$. The homogeneous solutions of eqs. (3.12) and (3.14) (that of eq. (3.13)) are given by $f_{J}^{(\mathrm{FP})}\left(f_{J}^{(\eta)}\right)$. For the case of $v \neq 0$, we also define the function $\widehat{f}_{J}^{(\eta)}$, obeying

$$
\left(\Delta_{J}-g^{2} v^{2}\right) \hat{f}_{J}^{(\eta)}=0,
$$

and

$$
\widehat{f}_{J}^{(\eta)}(r \rightarrow 0) \simeq r^{2 J}
$$

The function $\widehat{f}_{J}^{(\eta)}$ is given by

$$
\widehat{f}_{J}^{(\eta)}(r)=2^{2 J+1} \Gamma(2 J+2)(g v)^{-(2 J+1)} \frac{I_{2 J+1}(g v r)}{r},
$$

where $I_{2 J+1}$ is the modified Bessel function. Notice that, when $v=0$, we will not use the function $\widehat{f}_{J}^{(\eta)}$ and hence is not defined.

\section{3 $S$ and NG modes with $J=0$}

In this subsection, we consider the $S$ and NG modes with $J=0$. The fluctuation operator $\mathcal{M}_{J=0}^{(S, \varphi)}$ is in $2 \times 2$ form, and the solutions of the equation,

$$
\mathcal{M}_{J=0}^{(S, \varphi)} \Psi=0
$$

can be written as follows:

$$
\Psi \equiv\left(\begin{array}{c}
\Psi^{(\mathrm{top})} \\
\Psi^{(\mathrm{bot})}
\end{array}\right) \equiv\left(\begin{array}{c}
\partial_{r} \chi \\
g \bar{\phi} \chi
\end{array}\right)+\left(\begin{array}{c}
-2 \frac{\bar{\phi}^{\prime}}{g^{2} \bar{\phi}^{3}} \zeta \\
\frac{1}{g \bar{\phi}} \zeta
\end{array}\right),
$$

where the functions $\chi$ and $\zeta$ obey eq. (3.12) with $\eta=0$ and eq. (3.14), respectively.

As two independent solutions, we adopt the followings:

1. We take $\zeta_{1}=0$, and

$$
\chi_{1}(r)=1,
$$

which gives

$$
\Psi_{1}(r)=\left(\begin{array}{c}
0 \\
g \bar{\phi}
\end{array}\right) .
$$

2. We take

$$
\zeta_{2}(r)=1
$$

while $\chi_{2}(r \rightarrow 0) \sim O\left(r^{2}\right)$. Then,

$$
\Psi_{2}(r \rightarrow 0) \simeq\left(\begin{array}{c}
-\frac{\xi}{4} r \\
\frac{1}{g \bar{\phi}_{C}}
\end{array}\right)
$$


Consequently, we find

$$
\mathcal{D}_{0}^{(S, \varphi)}(r \rightarrow 0) \simeq \frac{1}{4} \xi g \bar{\phi}_{C} r
$$

\section{Functional determinants: case with $v \neq 0$}

Now, we are at the position to discuss the decay rate of the false vacuum. In this section, we consider the case with $v \neq 0$. Choosing the false vacuum as $\left(A_{\mu}, \Phi\right)_{\text {false vacuum }}=(0, v / \sqrt{2})$, the bounce solution is uniquely determined:

$$
\left(A_{\mu}, \Phi\right)_{\text {bounce }}=(0, \bar{\phi} / \sqrt{2}) .
$$

As we show in eq. (2.25), the prefactor $\mathcal{A}$ is given by the product of the functional determinants of the fluctuation operators. In the following, we discuss each contribution separately.

\section{1 $v \neq 0$ : contribution of $J \neq 0$}

Let us discuss the behavior of the functions $\Psi_{I}$ at $r \rightarrow \infty$. The behavior of $\chi_{I}, \eta_{I}$, and $\zeta_{I}$ can be understood by using the fact that $\bar{\phi}^{\prime}$ is exponentially suppressed at $r \rightarrow \infty$.

1. Because $\chi_{1}(r)=r^{2 J}$,

$$
\Psi_{1}(r \rightarrow \infty) \simeq\left(\begin{array}{c}
2 J r^{2 J-1} \\
L r^{2 J-1} \\
g v r^{2 J}
\end{array}\right)
$$

2. Because $\chi_{2}$ is given by the sum of a homogeneous solution and a particular solution (which we denote $\delta \chi^{(\eta)}$ ), the second set of the mode functions can be expressed as

$$
\begin{aligned}
& \chi_{2}(r)=a_{1} r^{2 J}+\delta \chi^{(\eta)}(r), \\
& \eta_{2}(r)=f_{J}^{(\eta)}(r)
\end{aligned}
$$

where $a_{1}$ is a constant. (Here and hereafter, dumping modes are neglected.) The function $\delta \chi^{(\eta)}$ satisfies the following equation:

$$
\Delta_{J} \delta \chi^{(\eta)}=\frac{2 \bar{\phi}^{\prime}}{r g^{2} \bar{\phi}^{3}} f_{J}^{(\eta)}
$$

We eliminate a term proportional to $r^{2 J}$ in $\delta \chi^{(\eta)}$ with the redefinition of $a_{1}$. Then, at $r \rightarrow \infty, \delta \chi^{(\eta)}$ behaves as

$$
\delta \chi^{(\eta)}(r \rightarrow \infty) \simeq-\frac{2 m_{\phi} \kappa}{g^{2} v^{3}\left(g v-m_{\phi}\right)^{2} r^{5 / 2}} e^{-m_{\phi} r} f_{J}^{(\eta)}+\cdots .
$$


(In appendix B, we discuss a procedure to derive asymptotic behavior of the solutions of the differential equation of this type.) Thus, the asymptotic behavior of $\Psi_{2}$ is given by

$$
\Psi_{2}(r \rightarrow \infty) \simeq a_{1} \Psi_{1}(r \rightarrow \infty)+\left(\begin{array}{c}
O\left(r^{-1} f_{J}^{(\eta)}\right) \\
\frac{1}{L g^{2} v^{2}} \partial_{r} f_{J}^{(\eta)} \\
O\left(r^{-5 / 2} e^{-m_{\phi} r} f_{J}^{(\eta)}\right)
\end{array}\right)
$$

3. For $\Psi_{3}$, we denote

$$
\begin{aligned}
& \chi_{3}(r)=b_{1} r^{2 J}+b_{2} \delta \chi^{(\eta)}(r)+\delta \chi^{(\zeta)}(r), \\
& \eta_{3}(r)=b_{2} f_{J}^{(\eta)}(r)+\delta \eta^{(\zeta)}(r), \\
& \zeta_{3}(r)=r^{2 J}
\end{aligned}
$$

with $b_{1}$ and $b_{2}$ being constants. The functions $\delta \chi^{(\zeta)}$ and $\delta \eta^{(\zeta)}$ obey the following equations:

$$
\begin{aligned}
& \Delta_{J} \delta \chi^{(\zeta)}=\frac{2 \bar{\phi}^{\prime}}{r g^{2} \bar{\phi}^{3}} \delta \eta^{(\zeta)}+\frac{2}{r^{3}} \partial_{r}\left(\frac{\bar{\phi}^{\prime}}{g^{2} \bar{\phi}^{3}} r^{2 J+3}\right)-\xi r^{2 J} \\
& \left(\Delta_{J}-g^{2} \bar{\phi}^{2}\right) \delta \eta^{(\zeta)}-\frac{2 \bar{\phi}^{\prime}}{r^{2} \bar{\phi}} \partial_{r}\left(r^{2} \delta \eta^{(\zeta)}\right)=-\frac{2 L^{2} \bar{\phi}^{\prime}}{\bar{\phi}} r^{2 J-1}
\end{aligned}
$$

and they asymptotically behave as

$$
\begin{aligned}
\delta \chi^{(\zeta)}(r \rightarrow \infty) & \simeq-\frac{1}{8(J+1)} \xi r^{2 J+2}, \\
\delta \eta^{(\zeta)}(r \rightarrow \infty) & \simeq 0 .
\end{aligned}
$$

Then, we obtain

$$
\Psi_{3}(r \rightarrow \infty) \simeq b_{1} \Psi_{1}(r \rightarrow \infty)+b_{2} \Psi_{2}(r \rightarrow \infty)-\frac{\xi}{8(J+1)}\left(\begin{array}{c}
(2 J+2) r^{2 J+1} \\
L r^{2 J+1} \\
g v r^{2 J+2}
\end{array}\right)
$$

Consequently, the determinant defined in eq. (3.17) behaves as

$$
\mathcal{D}_{J}^{(S, L, \varphi)}(r \rightarrow \infty) \sim \operatorname{det}\left(\begin{array}{ccc}
O\left(r^{2 J-1}\right) & O\left(r^{-1} f_{J}^{(\eta)}\right) & O\left(r^{2 J+1}\right) \\
O\left(r^{2 J-1}\right) & O\left(f_{J}^{(\eta)}\right) & O\left(r^{2 J+1}\right) \\
O\left(r^{2 J}\right) & O\left(r^{-5 / 2} e^{-m_{\phi} r} f_{J}^{(\eta)}\right) & O\left(r^{2 J+2}\right)
\end{array}\right)
$$

and

$$
\mathcal{D}_{J}^{(S, L, \varphi)}(r \rightarrow \infty) \simeq \frac{J \xi}{L^{3}} f_{J}^{(\eta)} r^{4 J+1} .
$$

For the calculation of the decay rate of the false vacuum, we also need the functional determinants around the false vacuum. Notice that, in the argument so far, we have only 
used the fact that $\bar{\phi}$ is a solution of the classical equation of motion and is non-vanishing. Thus, three independent solutions of eq. (3.10) can be obtained by the same argument but replacing $\bar{\phi} \rightarrow v$. (Notice that $\Phi=v / \sqrt{2}$ is also a solution of the classical equation of motion.) We find

$$
\frac{\mathcal{D}_{J}^{(S, L, \varphi)}(r \rightarrow 0)}{\widehat{\mathcal{D}}_{J}^{(S, L, \varphi)}(r \rightarrow 0)}=\frac{v}{\bar{\phi}_{C}}
$$

and

$$
\frac{\mathcal{D}_{J}^{(S, L, \varphi)}(r \rightarrow \infty)}{\widehat{\mathcal{D}}_{J}^{(S, L, \varphi)}(r \rightarrow \infty)}=\frac{f_{J}^{(\eta)}}{\widehat{f}_{J}^{(\eta)}}
$$

leading to

$$
\frac{\operatorname{Det} \mathcal{M}_{J}^{(S, L, \varphi)}}{\operatorname{Det} \widehat{\mathcal{M}}_{J}^{(S, L, \varphi)}}=\frac{\bar{\phi}_{C} f_{J}^{(\eta)}\left(r_{\infty}\right)}{v \widehat{f}_{J}^{(\eta)}\left(r_{\infty}\right)} .
$$

$4.2 v \neq 0$ : contribution of $J=0$

The case of $J=0$ is similar to that for $J \neq 0$. Thus, we just show the results. Using eq. (3.39),

$$
\frac{\mathcal{D}_{0}^{(S, \varphi)}(r \rightarrow 0)}{\widehat{\mathcal{D}}_{0}^{(S, \varphi)}(r \rightarrow 0)}=\frac{\bar{\phi}_{C}}{v} .
$$

In addition, the asymptotic behavior of $\Psi_{I}$ (with $I=1$ and 2 ) is

$$
\begin{aligned}
& \Psi_{1}(r \rightarrow \infty) \simeq\left(\begin{array}{c}
0 \\
g v
\end{array}\right), \\
& \Psi_{2}(r \rightarrow \infty) \simeq b_{1} \Psi_{1}(r \rightarrow \infty)-\frac{1}{8} \xi\left(\begin{array}{c}
2 r \\
g v r^{2}
\end{array}\right),
\end{aligned}
$$

with $b_{1}$ being a constant, and hence

$$
\frac{\mathcal{D}_{J}^{(S, L, \varphi)}(r \rightarrow \infty)}{\widehat{\mathcal{D}}_{J}^{(S, L, \varphi)}(r \rightarrow \infty)}=1 .
$$

Consequently,

$$
\frac{\operatorname{Det} \mathcal{M}_{0}^{(S, \varphi)}}{\operatorname{Det} \widehat{\mathcal{M}}_{0}^{(S, \varphi)}}=\frac{v}{\bar{\phi}_{C}}
$$

\section{$4.3 v \neq 0$ : final result}

Combining the contributions of $J=0$ and $J \neq 0$, we obtain

$$
\mathcal{A}^{(S, L, \varphi)}=\left(\frac{\bar{\phi}_{C}}{v}\right)^{1 / 2} \prod_{J \geq 1 / 2}\left[\frac{\bar{\phi}_{C} f_{J}^{(\eta)}\left(r_{\infty}\right)}{v \widehat{f}_{J}^{(\eta)}\left(r_{\infty}\right)}\right]^{-(2 J+1)^{2} / 2} .
$$


The above expression is manifestly $\xi$-independent. In addition, the other contributions are given by

$$
\begin{aligned}
\mathcal{A}^{(T)} & =\prod_{J=1 / 2}^{\infty}\left[\frac{f_{J}^{(T)}\left(r_{\infty}\right)}{\widehat{f}_{J}^{(T)}\left(r_{\infty}\right)}\right]^{-(2 J+1)^{2}}, \\
\mathcal{A}^{(\bar{c}, c)} & =1 .
\end{aligned}
$$

When $v \neq 0, \widehat{f}_{J}^{(\eta)}\left(r_{\infty}\right) \simeq \widehat{f}_{J}^{(\infty)}(r ; g v)$, and $\widehat{f}_{J}^{(T)}\left(r_{\infty}\right) \simeq \widehat{f}_{J}^{(\infty)}(r ; g v)$, where

$$
\widehat{f}_{J}^{(\infty)}(r ; m) \equiv \sqrt{\frac{2}{\pi}} \Gamma(2 J+2)\left(\frac{m}{2}\right)^{-2 J} \frac{e^{m r}}{(m r)^{3 / 2}} .
$$

Notice that the contribution of each angular momentum $J$ is finite.

The above result can be compared with that with another gauge fixing function, $\mathcal{F}^{\left(R_{\xi}\right)}=\partial_{\mu} A_{\mu}-2 \xi g(\operatorname{Re} \Phi)(\operatorname{Im} \Phi)$, which has been used in literature. For $v \neq 0$, the calculation with $\mathcal{F}^{\left(R_{\xi}\right)}$ has been performed in [6]. We can see that the two analyses give the same expression for the decay rate of the false vacuum.

\section{Functional determinants: case with $v=0$}

Next, we discuss the case in which the U(1) symmetry is unbroken in the false vacuum, i.e., $v=0$. As discussed in section 2, when $v=0$, there exists a class of bounce (i.e., the solution of the classical equation of motion with relevant boundary condition). All the bounce configurations are related by the global $\mathrm{U}(1)$ transformation which is unbroken at the false vacuum. We parameterize the bounce configurations for the gauge fixing function given in eq. (2.10) as

$$
\left(A_{\mu}, \Phi\right)_{\text {bounce }}=\left(0, \bar{\phi} e^{i \vartheta} / \sqrt{2}\right)
$$

where $0 \leq \vartheta<2 \pi$. We need to take account of all the bounce configurations in the calculation of the decay rate, as we will discuss below, which requires the integration over the variable $\vartheta$.

Importantly, expanding the gauge and scalar fields as eq. (2.14), the fluctuation operators are $\vartheta$-independent. This is an advantage of taking the gauge fixing function given in eq. (2.10), with which the integration over $\vartheta$ is easily performed.

\section{$5.1 v=0$ : contribution of $J \neq 0$}

In this subsection, we consider the contribution of $J \neq 0$ modes to $\mathcal{A}^{(S, L, \varphi)}$. Even for the case of $v=0$, eqs. (3.11)-(3.14) are useful to study the functional determinants.

The behavior of the function $\Psi_{I}$ around $r \rightarrow 0$ is insensitive to the value of $v$; thus, $\mathcal{D}_{J}^{(S, L, \varphi)}(r \rightarrow 0)$ is given in eq. (3.26) even for $v=0$. Next, we consider the asymptotic behavior of $\mathcal{D}_{J}^{(S, L, \varphi)}(r \rightarrow \infty)$. 
1. For the first solution $\Psi_{1}$, we are taking

$$
\chi_{1}(r \rightarrow \infty)=r^{2 J}
$$

and hence

$$
\Psi_{1}(r \rightarrow \infty) \simeq\left(\begin{array}{c}
2 J r^{2 J-1} \\
L r^{2 J-1} \\
0
\end{array}\right)
$$

2. For the second set of mode functions, $\chi_{2}$ and $\eta_{2}$ behave as

$$
\begin{aligned}
& \chi_{2}(r \rightarrow \infty) \simeq a_{1} r^{2 J}-\frac{1}{2 m_{\phi} g^{2} \bar{\phi}^{2} r} f_{J}^{(\eta)}+\cdots, \\
& \eta_{2}(r \rightarrow \infty)=f_{J}^{(\eta)}
\end{aligned}
$$

with $a_{1}$ being a constant. Here, we have used $f_{J}^{(\eta)}(r \rightarrow \infty) \propto r^{-2}$; this asymptotic behavior can be derived by using the fact that, in the left-hand side of eq. (3.27), the last term dominates when $r \rightarrow \infty$. One can see that the leading order contributions to the top and the middle components of $\Psi_{2}$ vanish. For the calculation of the top and middle components, it is convenient to use eqs. (3.15) and (3.16) to obtain

$$
\Psi_{2}(r \rightarrow \infty) \simeq a_{1} \Psi_{1}+\left(\begin{array}{c}
-\frac{r}{L^{2}} f_{J}^{(\eta)} \\
-\frac{2 r}{L^{3}} f_{J}^{(\eta)} \\
-\frac{1}{2 m_{\phi} g \bar{\phi} r} f_{J}^{(\eta)}
\end{array}\right)
$$

Notice that $\Psi_{2}^{(\text {bot })}(r \rightarrow \infty) \sim O\left(e^{m_{\phi} r} / r^{3 / 2}\right)$.

3. For $\Psi_{3}$, we find

$$
\begin{aligned}
& \chi_{3}(r \rightarrow \infty) \simeq b_{1} r^{2 J}-\frac{b_{2}}{2 m_{\phi} g^{2} \bar{\phi}_{C}^{2} r} f_{J}^{(\eta)}-\frac{1}{g^{2} \bar{\phi}^{2}} r^{2 J}+\cdots, \\
& \eta_{3}(r \rightarrow \infty) \simeq b_{2} f_{J}^{(\eta)}+2 J r^{2 J}+\cdots, \\
& \zeta_{3}(r \rightarrow \infty)=r^{2 J}
\end{aligned}
$$

with $b_{1}$ and $b_{2}$ being constants. Again, using eqs. (3.15) and (3.16),

$$
\Psi_{3}(r \rightarrow \infty) \simeq b_{1} \Psi_{1}+b_{2} \Psi_{2}+\left(\begin{array}{c}
-\frac{(J+1) \xi-J}{4(J+1)} r^{2 J+1} \\
-\frac{2 J[(J+1) \xi-(J+2)]}{4 L(J+1)} r^{2 J+1} \\
O\left(\bar{\phi} r^{2 J+2}\right)
\end{array}\right)
$$


Using eqs. (5.3), (5.6), and (5.10), the determinant is given by

$$
\mathcal{D}_{J}^{(S, L, \varphi)}(r \rightarrow \infty)=\frac{4 J^{2}[(J+1) \xi+J] r^{4 J-1} f_{J}^{(\eta)}}{2 L^{3} m_{\phi} g \bar{\phi}} .
$$

For the calculation of the functional determinants around the false vacuum, we find the following solutions of eq. (3.10):

$$
\begin{aligned}
& \widehat{\Psi}_{1}(r)=\left(\begin{array}{c}
2 J r^{2 J-1} \\
L r^{2 J-1} \\
0
\end{array}\right), \\
& \widehat{\Psi}_{2}(r)=\left(\begin{array}{c}
\frac{(J+1) \xi-J}{2 L^{2}} r^{2 J+1} \\
\frac{(J+1) \xi-(J+2)}{4 L(J+1)} r^{2 J+1} \\
0
\end{array}\right), \\
& \widehat{\Psi}_{3}(r)=\left(\begin{array}{c}
0 \\
0 \\
\widehat{f}_{J}^{(\sigma)}(r)
\end{array}\right) \cdot
\end{aligned}
$$

Here, the function $\widehat{f}_{J}^{(\sigma)}$ satisfies

$$
\left(\Delta_{J}-m_{\phi}^{2}\right) \widehat{f}_{J}^{(\sigma)}=0
$$

with

$$
\widehat{f}_{J}^{(\sigma)}(r \rightarrow 0) \simeq r^{2 J}
$$

The explicit form of $\widehat{f}_{J}^{(\sigma)}$ is given by

$$
\widehat{f}_{J}^{(\sigma)}(r)=2^{2 J+1} \Gamma(2 J+2) m_{\phi}^{-(2 J+1)} \frac{I_{2 J+1}\left(m_{\phi} r\right)}{r} .
$$

Then, we find

$$
\widehat{\mathcal{D}}_{J}^{(S, L, \varphi)}(r)=-\frac{2 J[(J+1) \xi+J]}{L^{3}} r^{4 J} \widehat{f}_{J}^{(\sigma)}(r) .
$$

Using eqs. (3.26), (5.11), and (5.18), we obtain

$$
\frac{\operatorname{Det} \mathcal{M}_{J}^{(S, L, \varphi)}}{\operatorname{Det} \widehat{\mathcal{M}}_{J}^{(S, L, \varphi)}}=\frac{J \bar{\phi}_{C}}{m_{\phi}} \frac{f_{J}^{(\eta)}\left(r_{\infty}\right)}{r_{\infty} \bar{\phi}\left(r_{\infty}\right) \widehat{f}_{J}^{(\sigma)}\left(r_{\infty}\right)} .
$$

Importantly, the above result is $\xi$-independent.

We also give an alternative expression of the ratio of $\operatorname{Det} \mathcal{M}_{J}^{(S, L, \varphi)}$ to $\operatorname{Det} \widehat{\mathcal{M}}_{J}^{(S, L, \varphi)}$, which is useful for the numerical calculation. Consider the limit of vanishing gauge coupling constant $g$. Even in such a limit, eq. (5.18) is still valid if we evaluate $f_{J}^{(\eta)}$ with $g=0$. 
In addition, when $g=0$, the fluctuation operator $\mathcal{M}_{J}^{(S, L, \varphi)}$ given in eq. (2.20) is blockdiagonal; the upper $2 \times 2$ part becomes independent of the bounce $\bar{\phi}$, leading to

$$
\left[\frac{\operatorname{Det} \mathcal{M}_{J}^{(S, L, \varphi)}}{\operatorname{Det} \widehat{\mathcal{M}}_{J}^{(S, L, \varphi)}}\right]_{g=0}=\frac{\operatorname{Det}\left[-\Delta_{J}+\left(\Delta_{0} \bar{\phi}\right) / \bar{\phi}\right]}{\operatorname{Det}\left(-\Delta_{J}+m_{\phi}^{2}\right)}
$$

Thus, the following relation holds:

$$
\frac{J \bar{\phi}_{C}}{m_{\phi}} \frac{\left[f_{J}^{(\eta)}\right]_{g=0}\left(r_{\infty}\right)}{r_{\infty} \bar{\phi}\left(r_{\infty}\right) \widehat{f}_{J}^{(\sigma)}\left(r_{\infty}\right)}=\frac{f_{J}^{(\sigma)}\left(r_{\infty}\right)}{\widehat{f}_{J}^{(\sigma)}\left(r_{\infty}\right)},
$$

where the function $\left[f_{J}^{(\eta)}\right]_{g=0}$ obeys

$$
\Delta_{J}\left[f_{J}^{(\eta)}\right]_{g=0}-\frac{2 \bar{\phi}^{\prime}}{r^{2} \bar{\phi}} \partial_{r}\left(r^{2}\left[f_{J}^{(\eta)}\right]_{g=0}\right)=0
$$

with

$$
\left[f_{J}^{(\eta)}\right]_{g=0}(r \rightarrow 0) \simeq r^{2 J}
$$

while $f_{J}^{(\sigma)}$ is the function which satisfies

$$
\Delta_{J} f_{J}^{(\sigma)}-\frac{\left(\Delta_{0} \bar{\phi}\right)}{\bar{\phi}} f_{J}^{(\sigma)}=0
$$

with

$$
f_{J}^{(\sigma)}(r \rightarrow 0) \simeq r^{2 J}
$$

Using eq. (5.21), the ratio of the functional determinants can be rewritten as

$$
\frac{\operatorname{Det} \mathcal{M}_{J}^{(S, L, \varphi)}}{\operatorname{Det} \widehat{\mathcal{M}}_{J}^{(S, L, \varphi)}}=\frac{f_{J}^{(\sigma)}\left(r_{\infty}\right)}{\widehat{f}_{J}^{(\sigma)}\left(r_{\infty}\right)} \frac{f_{J}^{(\eta)}\left(r_{\infty}\right)}{\left[f_{J}^{(\eta)}\right]_{g=0}\left(r_{\infty}\right)} .
$$

We note here that the evolution equations of the functions $\widehat{f}_{J}^{(\sigma)}$ and $f_{J}^{(\sigma)}$, which are given in eqs. (5.15) and (5.24), respectively, are asymptotically the same at $r \rightarrow \infty$; the same is true for the evolution equations of the functions $\left[f_{J}^{(\eta)}\right]_{g=0}$ and $f_{J}^{(\eta)}$. Thus, the ratios $f_{J}^{(\sigma)} / \widehat{f}_{J}^{(\sigma)}$ and $f_{J}^{(\eta)} /\left[f_{J}^{(\eta)}\right]_{g=0}$ converge to constant values in the limit of $r \rightarrow \infty$. Numerically, the right-hand side of eq. (5.26) converges much faster than that of eq. (5.19) at $r \rightarrow \infty$. Thus, eq. (5.26) is useful for the numerical calculation.

\section{$5.2 v=0:$ contribution of $J=0$}

Now, we consider the functional determinants of $\mathcal{M}_{J=0}^{(S, \varphi)}$ and $\widehat{\mathcal{M}}_{J=0}^{(S, \varphi)}$ for the case of $v=0$. When $v=0$, the $J=0$ mode requires special treatment because all the classical solutions given in eq. (2.13), parameterized by $\vartheta$, becomes the bounce and contributes to the decay rate. As a consequence, the fluctuation operator $\mathcal{M}_{J=0}^{(S, \varphi)}$ has a zero mode [10]. Because of the zero mode, $\operatorname{Det} \mathcal{M}_{J=0}^{(S, \varphi)}$ vanishes if one naively calculates the functional determinant, 
resulting in a divergent behavior of the decay rate of the false vacuum. In the following, we discuss how to calculate the $J=0$ contribution to obtain a meaningful result.

For the case of $J=0$, we need solutions of eq. (3.33). From eq. (3.12) (with $\eta=0$ ), the following equations can be derived:

$$
\begin{aligned}
& \Psi^{\text {(top })}=-\xi \frac{1}{r^{3}} \int_{0}^{r} d r_{1} r_{1}^{3} \zeta\left(r_{1}\right) \\
& \Psi^{(\mathrm{bot})}=-\xi g \bar{\phi}\left[c+\int^{r} d r_{1} \frac{1}{r_{1}^{3}} \int_{0}^{r_{1}} d r_{2} r_{2}^{3} \zeta\left(r_{2}\right)\right]+g \bar{\phi} \int^{r} d r_{1} \frac{1}{g^{2} \bar{\phi}^{2}\left(r_{1}\right)} \zeta^{\prime}\left(r_{1}\right),
\end{aligned}
$$

where $c$ is an arbitrary constant, and $\zeta^{\prime} \equiv \partial_{r} \zeta$. These equations are useful to derive the solutions of eq. (3.33). For $J=0$, the first solution of eq. (3.33) is obtained by taking $\chi_{1}(r)=1$ and $\zeta_{1}(r)=0$ :

$$
\Psi_{1}(r)=\left(\begin{array}{c}
0 \\
g \bar{\phi}
\end{array}\right) .
$$

The second solution is obtained with $\chi_{2}(0)=0$ and $\zeta_{2}(r)=1$ :

$$
\Psi_{2}(r)=\left(\begin{array}{c}
-\frac{1}{4} \xi r \\
-\frac{1}{8} \xi r^{2} g \bar{\phi}
\end{array}\right) .
$$

In addition, two independent solutions of $\widehat{\mathcal{M}}_{0}^{(S, \varphi)} \widehat{\Psi}=0$ are taken to be

$$
\begin{aligned}
& \widehat{\Psi}_{1}=\left(\begin{array}{c}
r \\
0
\end{array}\right), \\
& \widehat{\Psi}_{2}=\left(\begin{array}{c}
0 \\
\hat{f}_{0}^{(\sigma)}
\end{array}\right) .
\end{aligned}
$$

One can see that $\Psi_{1}(r \rightarrow \infty) \simeq 0$, and $\operatorname{Det} \mathcal{M}_{J=0}^{(S, \varphi)}=0$. This is a consequence of the zero-mode eigenfunction of $\mathcal{M}_{J=0}^{(S, \varphi)}$. Indeed, the following function:

$$
\Psi^{\text {(zero-mode) }}=\mathcal{N} \mathcal{Y}_{0,0,0}^{-1}\left(\begin{array}{l}
0 \\
\bar{\phi}
\end{array}\right),
$$

satisfies the conditions to be the zero-mode eigenfunction, i.e., $\mathcal{M}_{0}^{(S, \varphi)} \Psi^{\text {(zero-mode) }}=0$, and $\Psi^{\text {(zero-mode) }}(r \rightarrow \infty)=0$. Here, $\mathcal{N}$ is the normalization factor, given by ${ }^{3}$

$$
\mathcal{N}^{2} \int d^{4} r \bar{\phi}^{2}=2 \pi
$$

\footnotetext{
${ }^{3}$ We adopt the normalization of the mode functions so that the path integral is defined as $\prod_{n} d c^{(n)}$, with $c^{(n)}$ being the expansion coefficient of the wave function with respect to the mode functions: $\Psi=\sum_{n} c^{(n)} \Psi^{(n)}$. (In eq. (5.35), $d c^{\text {(zero-mode) }}$ is denoted as $\mathcal{D} \Psi^{\text {(zero-mode) }}$.) Some of the previous studies use different definition of the path integral as $\prod_{n}\left(d c^{(n)} / \sqrt{2 \pi}\right)$, with which the right-hand side of eq. (5.34) should be 1 .
} 
The zero mode stems from the global U(1) symmetry which is preserved in the false vacuum; such a $\mathrm{U}(1)$ symmetry relates the bounce configurations parameterized by $\vartheta$ (see eq. (5.1)). The zero mode given in eq. (5.33) is nothing but the mode generated by the global U(1) transformation of the bounce. Thus, the path integral of the zero mode should be understood as the integration over the bounce configurations related by the U(1) transformation. Based on this consideration, we can perform the following replacements [10]:

$$
\int \mathcal{D} \Psi^{\text {(zero-mode) }} \rightarrow \frac{1}{\mathcal{N}} \int_{0}^{2 \pi} d \vartheta
$$

where $\int \mathcal{D} \Psi^{\text {(zero-mode) }}$ denotes the path integral of the zero mode, and, using the fact that the fluctuation operators do not depend on $\vartheta$,

$$
\left[\frac{\operatorname{Det} \mathcal{M}_{0}^{(S, \varphi)}}{\operatorname{Det} \widehat{\mathcal{M}}_{0}^{(S, \varphi)}}\right]^{-1 / 2} \rightarrow \frac{2 \pi}{\mathcal{N}}\left[\frac{\operatorname{Det}^{\prime} \mathcal{M}_{0}^{(S, \varphi)}}{\operatorname{Det} \widehat{\mathcal{M}}_{0}^{(S, \varphi)}}\right]^{-1 / 2},
$$

where Det' implies that the zero eigenvalue is omitted from the functional determinant.

The zero eigenvalue can be omitted with the use of the following modified fluctuation operator:

$$
\mathcal{M}_{0}^{(S, \varphi)}+\operatorname{diag}(\nu, \nu)
$$

where $\nu$ is a (small) constant. Each eigenfunction of $\mathcal{M}_{0}^{(S, \varphi)}$ is also an eigenfunction of the above modified fluctuation operator; the eigenvalue increases by $\nu$. Especially, $\Psi^{\text {(zero-mode) }}$ given in eq. (5.33) is an eigenfunction of the above modified fluctuation operator with the eigenvalue of $\nu$. Thus, we eliminate the zero eigenvalue from $\operatorname{Det} \mathcal{M}_{0}^{(S, \varphi)}$ as

$$
\frac{\operatorname{Det}^{\prime} \mathcal{M}_{0}^{(S, \varphi)}}{\operatorname{Det} \widehat{\mathcal{M}}_{0}^{(S, \varphi)}}=\lim _{\nu \rightarrow 0} \frac{1}{\nu} \frac{\operatorname{Det}\left[\mathcal{M}_{0}^{(S, \varphi)}+\operatorname{diag}(\nu, \nu)\right]}{\operatorname{Det} \widehat{\mathcal{M}}_{0}^{(S, \varphi)}}
$$

For the calculation of the functional determinant of the modified fluctuation operator, we solve the following equation:

$$
\left[\mathcal{M}_{0}^{(S, \varphi)}+\operatorname{diag}(\nu, \nu)\right] \Psi^{(\nu)}=0
$$

with the condition $\lim _{\nu \rightarrow 0} \Psi^{(\nu)}=\Psi_{1}$. Defining

$$
\mathcal{D}_{0}^{\prime(S, \varphi)}(r)=\lim _{\nu \rightarrow 0} \frac{\operatorname{det}\left(\Psi^{(\nu)}(r) \Psi_{2}(r)\right)}{\nu},
$$

we obtain

$$
\frac{\operatorname{Det}^{\prime} \mathcal{M}_{0}^{(S, \varphi)}}{\operatorname{Det} \widehat{\mathcal{M}}_{0}^{(S, \varphi)}}=\left[\frac{\mathcal{D}_{0}^{(S, \varphi)}(r \rightarrow 0)}{\widehat{\mathcal{D}}_{0}^{(S, \varphi)}(r \rightarrow 0)}\right]^{-1} \frac{\mathcal{D}_{0}^{(S, \varphi)}\left(r_{\infty}\right)}{\widehat{\mathcal{D}}_{0}^{(S, \varphi)}\left(r_{\infty}\right)}
$$

For the calculation of $\Psi^{(\nu)}$ up to $O(\nu)$, we expand $\Psi^{(\nu)}$ as

$$
\Psi^{(\nu)}=\Psi_{1}+\nu \check{\Psi}+O\left(\nu^{2}\right)
$$


with which

$$
\mathcal{D}_{0}^{\prime(S, \varphi)}\left(r_{\infty}\right)=\operatorname{det}\left(\check{\Psi}\left(r_{\infty}\right) \Psi_{2}\left(r_{\infty}\right)\right)
$$

Here, $\check{\Psi}$ should satisfy

$$
\mathcal{M}_{0}^{(S, \varphi)} \check{\Psi}=-\left(\begin{array}{c}
0 \\
g \bar{\phi}
\end{array}\right) .
$$

The solution of the above equation is given in the following form:

$$
\check{\Psi}=\left(\begin{array}{c}
\check{\Psi}^{(\mathrm{top})} \\
\check{\Psi}^{(\mathrm{bot})}
\end{array}\right) \equiv\left(\begin{array}{c}
\partial_{r} \check{\chi} \\
g \bar{\phi} \check{\chi}
\end{array}\right)+\left(\begin{array}{c}
-2 \frac{\bar{\phi}^{\prime}}{g^{2} \bar{\phi}^{3}} \check{\zeta} \\
\frac{1}{g \bar{\phi}} \check{\zeta}
\end{array}\right),
$$

with the functions $\check{\chi}$ and $\check{\zeta}$ obeying

$$
\begin{aligned}
\Delta_{0} \check{\chi} & =\frac{2}{r^{3}} \partial_{r}\left(\frac{r^{3} \bar{\phi}^{\prime}}{g^{2} \bar{\phi}^{3}} \check{\zeta}\right)-\xi \check{\zeta}, \\
\Delta_{0} \check{\zeta} & =g^{2} \bar{\phi}^{2} .
\end{aligned}
$$

Notice that, from eq. (5.46), $\check{\zeta}$ is given by

$$
\check{\zeta}=\int_{0}^{r} d r_{1} r_{1}^{-3} \int_{0}^{r_{1}} d r_{2} r_{2}^{3} g^{2} \bar{\phi}^{2}\left(r_{2}\right)
$$

and hence $\check{\zeta}(r \rightarrow \infty)$ is a constant. Notably, $\check{\Psi}^{(\text {top })}$ and $\check{\Psi}^{(\text {bot })}$ satisfy similar equations as eqs. (5.27) and (5.28), respectively:

$$
\begin{aligned}
& \check{\Psi}^{(\mathrm{top})}(r)=-\xi \frac{1}{r^{3}} \int_{0}^{r} d r_{1} r_{1}^{3} \check{\zeta}\left(r_{1}\right), \\
& \check{\Psi}^{(\mathrm{bot})}(r)=-\xi g \bar{\phi} \int^{r} d r_{1} \frac{1}{r_{1}^{3}} \int_{0}^{r_{1}} d r_{2} r_{2}^{3} \check{\zeta}\left(r_{2}\right)+g \bar{\phi} \int^{r} d r_{1} \frac{1}{g^{2} \bar{\phi}^{2}\left(r_{1}\right)} \check{\zeta}^{\prime}\left(r_{1}\right) .
\end{aligned}
$$

We are interested in their behaviors at $r \rightarrow \infty$; in such a limit, (i) $\check{\Psi}^{(\mathrm{top})}$ is proportional to $r$ because $\check{\zeta}(r \rightarrow \infty)$ is a constant, and (ii) the asymptotic behavior of $\check{\Psi}^{(\text {bot })}$ is obtained from the fact that the first term of the right-hand side of eq. (5.49) vanishes when $r \rightarrow \infty$ and that $\check{\zeta}^{\prime}(r \rightarrow \infty) \simeq g^{2} / \pi \mathcal{N}^{2} r^{3}$. Remembering that $\bar{\phi}(r \rightarrow \infty)$ is approximately proportional to $e^{-m_{\phi} r} / r^{3 / 2}$, the asymptotic behavior of $\check{\Psi}$ is found to be

$$
\check{\Psi}(r \rightarrow \infty) \simeq\left(\begin{array}{c}
-\frac{1}{4} \xi r \check{\zeta} \\
\frac{g}{2 \pi \mathcal{N}^{2} m_{\phi} r^{3} \bar{\phi}}
\end{array}\right)
$$

Consequently, we obtain

$$
\left[\frac{\operatorname{Det} \mathcal{M}_{0}^{(S, \varphi)}}{\operatorname{Det} \widehat{\mathcal{M}}_{0}^{(S, \varphi)}}\right]^{-1 / 2} \rightarrow 2 \pi\left[\frac{1}{2 \pi m_{\phi} \bar{\phi}_{C} r_{\infty}^{3} \bar{\phi}\left(r_{\infty}\right) \widehat{f}_{0}^{(\sigma)}\left(r_{\infty}\right)}\right]^{-1 / 2}
$$

where we have used $f_{0}^{(\sigma)}(0)=1$. Notice that $\widehat{f}_{0}^{(\sigma)}(r \rightarrow \infty) \propto e^{m_{\phi} r} / r^{3 / 2}$, and that the above quantity is finite. 


\section{$5.3 v=0$ : final result}

The contributions of the $S, L$, and NG modes are

$$
\mathcal{A}^{(S, L, \varphi)}=2 \pi\left[2 \pi m_{\phi} \bar{\phi}_{C} r_{\infty}^{3} \bar{\phi}\left(r_{\infty}\right) \widehat{f}_{0}^{(\sigma)}\left(r_{\infty}\right)\right]^{1 / 2} \prod_{J \geq 1 / 2}\left[\frac{J \bar{\phi}_{C}}{m_{\phi}} \frac{f_{J}^{(\eta)}\left(r_{\infty}\right)}{r_{\infty} \bar{\phi}\left(r_{\infty}\right) \widehat{f}_{J}^{(\sigma)}\left(r_{\infty}\right)}\right]^{-(2 J+1)^{2} / 2},
$$

while the other contributions are

$$
\begin{aligned}
\mathcal{A}^{(T)} & =\prod_{J=1 / 2}^{\infty}\left[\frac{f_{J}^{(T)}\left(r_{\infty}\right)}{\widehat{f}_{J}^{(T)}\left(r_{\infty}\right)}\right]^{-(2 J+1)^{2}}, \\
\mathcal{A}^{(\bar{c}, c)} & =1 .
\end{aligned}
$$

For the case of $v=0, \widehat{f}_{J}^{(\eta)}\left(r_{\infty}\right) \propto r^{-2}$ and $\widehat{f}_{J}^{(T)}\left(r_{\infty}\right) \propto r^{2 J}$. We emphasize that the final result is $\xi$-independent.

Before closing this section, we comment on the calculation based on the $R_{\xi}$-like gauge fixing function, $\mathcal{F}^{\left(R_{\xi}\right)}=\partial_{\mu} A_{\mu}-2 \xi g(\operatorname{Re} \Phi)(\operatorname{Im} \Phi)$. As we have mentioned, in the case of $v=0$, there exists a class of bounce configuration which depends on the function $\Theta(r)$ obeying eq. (2.9); the function $\Theta$ is determined by its value at the origin, $\Theta(0)$. One technical difficulty is that the bounce configurations, as well as the fluctuation operators around the bounce, depend on $\Theta(0)$. If we adopt $\mathcal{F}^{\left(R_{\xi}\right)}$, we need to calculate the functional determinants as functions of $\Theta(0)$, and somehow integrate over $\Theta(0)$. Such an analysis is beyond the scope of this paper, because we have shown that the final result can be obtained with the use of the gauge fixing function $\mathcal{F}=\partial_{\mu} A_{\mu}$. Just for a comparison, we have calculated the functional determinants around the bounce configuration with $\Theta(0)=0$ (which results in $\Theta=0$ ), adopting $\mathcal{F}^{\left(R_{\xi}\right)}$. Based on the calculation with angular-momentum decomposition, we have checked that the contributions from the modes with $J \neq 0$ agree with the results of the present calculation. However, the contribution of the $J=0$ mode is hardly compared with our present result because the measure for the integration over $\Theta(0)$ is unknown. Notice that a hasty substitution of $\operatorname{Det}^{\prime}\left[\mathcal{M}_{0}^{(S, \varphi)}\right]_{R_{\xi}} / \operatorname{Det}\left[\widehat{\mathcal{M}}_{0}^{(S, \varphi)}\right]_{R_{\xi}}$ for $\Theta=0$ into eq. (5.36) will give a gauge dependent result, where $\left[\mathcal{M}_{0}^{(S, \varphi)}\right]_{R_{\xi}}$ and $\left[\widehat{\mathcal{M}}_{0}^{(S, \varphi)}\right]_{R_{\xi}}$ are fluctuation operators based on the $R_{\xi}$-like gauge.

\section{Renormalization}

So far, we have calculated the functional determinants by integrating out the field fluctuations around the bounce configuration and also around the false vacuum. Because these quantities are divergent [8], the renormalization is necessary to make the decay rate finite. In this section, we outline how to perform the renormalization. As in the previous sections, we pay particular attention to the effects of the gauge bosons and NG boson.

First, for notational simplicity, we introduce

$$
\delta \bar{\phi}^{2} \equiv \bar{\phi}^{2}-v^{2}
$$


and

$$
\delta \Omega \equiv \Omega-\widehat{\Omega}
$$

with

$$
\Omega \equiv \frac{\left(\partial^{2} \bar{\phi}\right)}{\bar{\phi}}+g^{2} \bar{\phi}^{2}
$$

Here, $\widehat{\Omega}$ is the value of $\Omega$ around the false vacuum (and hence is a constant):

$$
\widehat{\Omega}=\left\{\begin{array}{ll}
g^{2} v^{2} & : v \neq 0 \\
m_{\phi}^{2} & : v=0
\end{array} .\right.
$$

The calculation of the functional determinant of $\mathcal{M}^{\left(A_{\mu}, \varphi\right)}$ can be performed by treating $\delta \bar{\phi}^{2}$ and $\delta \Omega$ as perturbations; $\mathcal{M}^{\left(A_{\mu}, \varphi\right)}$ is given by the sum of the terms with different numbers of the insertions of $\delta \bar{\phi}^{2}$ and $\delta \Omega$.

Because we are interested in renormalizable theories, all the divergences are related to operators with mass dimension 4 or smaller. In the present model, such divergences show up at finite orders of the gauge or quartic scalar couplings at the one-loop level. In other words, the divergences are with limited numbers of the insertions of $\delta \bar{\phi}^{2}$ and $\delta \Omega$. The procedure to obtain renormalized decay rate is to calculate the functional determinant without the divergent part, which will be defined below, by the method adopted in the previous sections. The divergent part is separately calculated with the dimensional regularization using ordinary Feynman rules, and is made finite with the $\overline{\mathrm{MS}}$ subtraction.

The divergent part can be obtained by expanding the functional determinant with respect to $\delta \bar{\phi}^{2}$ and $\delta \Omega$, and keeping the terms corresponding to operators with mass dimensions less than or equal to 4 . Importantly, the divergent part should be properly subtracted from the functional determinant for each $J$. It may be performed with the fluctuation operators given in eqs. (2.20) and (2.22), which are obtained from the gauge fixing function of our choice. However, the calculation can be made easier if we use the fluctuation operators obtained from the $R_{\xi}$-like gauge fixing function; it is allowed because we have confirmed that the results of the calculations based on the two different gauge fixing functions agree with each other at least for $J \neq 0[6]$. Hereafter, we use these facts to simplify our calculation.

With a straightforward calculation, the divergent part of $\ln \left[\operatorname{Det} \mathcal{M}^{\left(A_{\mu}, \varphi\right)} / \operatorname{Det} \widehat{\mathcal{M}}^{\left(A_{\mu}, \varphi\right)}\right]^{-1 / 2}$ is obtained as

$$
\begin{aligned}
\delta \mathcal{S}_{\mathrm{div}}^{\left(A_{\mu}, \varphi\right)} \equiv & \operatorname{Tr}\left[g^{2} \delta \bar{\phi}^{2} \frac{1}{-\partial^{2}+g^{2} v^{2}}\right]-\frac{1}{2} \operatorname{Tr}\left[g^{2} \delta \bar{\phi}^{2} \frac{1}{-\partial^{2}+g^{2} v^{2}} g^{2} \delta \bar{\phi}^{2} \frac{1}{-\partial^{2}+g^{2} v^{2}}\right] \\
& +\frac{1}{2} \operatorname{Tr}\left[\delta \Omega \frac{1}{-\partial^{2}+\widehat{\Omega}}\right]-\frac{1}{4} \operatorname{Tr}\left[\delta \Omega \frac{1}{-\partial^{2}+\widehat{\Omega}} \delta \Omega \frac{1}{-\partial^{2}+\widehat{\Omega}}\right] \\
& -2 \operatorname{Tr}\left[\left(g \partial_{\mu} \bar{\phi}\right) \frac{1}{-\partial^{2}+g^{2} v^{2}}\left(g \partial_{\mu} \bar{\phi}\right) \frac{1}{-\partial^{2}+\widehat{\Omega}}\right],
\end{aligned}
$$


which means

$$
\ln \left[\frac{\operatorname{Det} \mathcal{M}^{\left(A_{\mu}, \varphi\right)}}{\operatorname{Det} \widehat{\mathcal{M}}^{\left(A_{\mu}, \varphi\right)}}\right]^{-1 / 2}+\delta \mathcal{S}_{\operatorname{div}}^{\left(A_{\mu}, \varphi\right)}=(\text { finite }) .
$$

As we see below, $\delta \mathcal{S}_{\mathrm{div}}^{\left(A_{\mu}, \varphi\right)}$ can be used to subtract the divergences with a relevant renormalization scheme (like the $\overline{\mathrm{MS}}$ scheme). Notice that the divergent part of the functional determinant of our interest is $\xi$-independent, and hence the decay rate of the false vacuum is gauge invariant even after the renormalization.

Hereafter, we calculate $\delta \mathcal{S}_{\text {div }}^{\left(A_{\mu}, \varphi\right)}$ with two different procedures. One is a decomposition with respect to the angular momentum, which is based on the following equality:

$$
\delta \mathcal{S}_{\operatorname{div}}^{\left(A_{\mu}, \varphi\right)}=\delta \mathcal{S}_{\operatorname{div}}^{(S, L, \varphi)}+\delta \mathcal{S}_{\operatorname{div}}^{(T)}
$$

where

$$
\begin{aligned}
& \delta \mathcal{S}_{\text {div }}^{(S, L, \varphi)} \equiv \frac{1}{2}\left[\ln \frac{\operatorname{Det}\left(\widehat{\mathcal{M}}_{R_{\xi=1}}^{\left(A_{\mu}, \varphi\right)}+\delta \mathcal{M}\right)}{\operatorname{Det} \widehat{\mathcal{M}}_{R_{\xi=1}}^{\left(A_{\mu}, \varphi\right)}}\right]_{(\delta \mathcal{M})^{2}}-2\left[\ln \frac{\operatorname{Det}\left(-\partial^{2}+g^{2} v^{2}+g^{2} \delta \bar{\phi}^{2}\right)}{\operatorname{Det}\left(-\partial^{2}+g^{2} v^{2}\right)}\right]_{\left(\delta \bar{\phi}^{2}\right)^{2}}, \\
& \delta \mathcal{S}_{\mathrm{div}}^{(T)} \equiv\left[\ln \frac{\operatorname{Det}\left(-\partial^{2}+g^{2} v^{2}+g^{2} \delta \bar{\phi}^{2}\right)}{\operatorname{Det}\left(-\partial^{2}+g^{2} v^{2}\right)}\right]_{\left(\delta \bar{\phi}^{2}\right)^{2}},
\end{aligned}
$$

with

$$
\begin{aligned}
\widehat{\mathcal{M}}_{R_{\xi=1}}^{\left(A_{\mu}, \varphi\right)} & \equiv\left(\begin{array}{cc}
\left(-\partial^{2}+g^{2} v^{2}\right) \delta_{\mu \nu} & 0 \\
0 & -\partial^{2}+\widehat{\Omega}
\end{array}\right), \\
\delta \mathcal{M} & \equiv\left(\begin{array}{cc}
g^{2} \delta \bar{\phi}^{2} \delta_{\mu \nu} & 2 g\left(\partial_{\nu} \bar{\phi}\right) \\
2 g\left(\partial_{\mu} \bar{\phi}\right) & \delta \Omega
\end{array}\right) .
\end{aligned}
$$

Here, $[\cdots]_{\mathcal{P}^{N}}$ indicates that the quantity in the square bracket is evaluated up to $O\left(\mathcal{P}^{N}\right)$. We note that $\widehat{\mathcal{M}}_{R_{\xi=1}}^{\left(A_{\mu}, \varphi\right)}$ is the fluctuation operator of $A_{\mu}$ and $\varphi$ in the $R_{\xi}$-like gauge with $\xi=1$ around the false vacuum. In addition, $\widehat{\mathcal{M}}_{R_{\xi=1}}^{\left(A_{\mu}, \varphi\right)}+\delta \mathcal{M}$ is the one around the bounce configuration with $\Theta(r)=0$. Because the results based on our choice of the gauge fixing and the $R_{\xi}$-like gauge fixing give the same result, eqs. (6.8) and (6.9) properly take account of the divergent part for each $J$. With eq. (2.35), the right-hand sides of eqs. (6.8) and (6.9) can be evaluated. The result is given as the sum of the contribution from each angular momentum. We denote

$$
\begin{aligned}
\delta \mathcal{S}_{\operatorname{div}}^{(S, L, \varphi)} & \equiv \sum_{J=0}^{\infty} s_{J}^{(S, L, \varphi)}, \\
\delta \mathcal{S}_{\operatorname{div}}^{(T)} & \equiv \sum_{J=0}^{\infty} s_{J}^{(T)} .
\end{aligned}
$$

Notice that, comparing eq. (6.9) with eq. (2.23), we can see that $\ln \mathcal{A}^{(T)}+\delta \mathcal{S}_{\text {div }}^{(T)}$ is finite. Thus, $\ln \mathcal{A}^{(S, L, \varphi)}+\delta \mathcal{S}_{\text {div }}^{(S, L, \varphi)}$ also is. A prescription for the calculation of the counter terms 
for each angular momentum, i.e., $s_{J}^{(S, L, \varphi)}$ and $s_{J}^{(T)}$, is given in appendix C (see eqs. (C.17) and (C.18)).

The quantity $\mathcal{S}_{\text {div }}^{\left(A_{\mu}, \varphi\right)}$ is also calculated with ordinary Feynman rules. The result is divergent; using the dimensional regularization based on $D$-dimensional theory, $\mathcal{S}_{\text {div }}^{\left(A_{\mu}, \varphi\right)}$ constrains a term proportional to $\bar{\epsilon}^{-1} \equiv \frac{2}{4-D}-\gamma+\ln 4 \pi$ (with $\gamma$ here being the Eular's constant). Such a term is exactly cancelled out by the counter term in the $\overline{\mathrm{MS}}$-scheme. We define $\delta \mathcal{S}_{\overline{\mathrm{MS}}}^{\left(A_{\mu}, \varphi\right)}$ from $\delta \mathcal{S}_{\mathrm{div}}^{\left(A_{\mu}, \varphi\right)}$ via the $\overline{\mathrm{MS}}$-subtraction:

$$
\left.\delta \mathcal{S}_{\overline{\mathrm{MS}}}^{\left(A_{\mu}, \varphi\right)} \equiv \delta \mathcal{S}_{\mathrm{div}}^{\left(A_{\mu}, \varphi\right)}\right|_{\overline{\mathrm{MS}} \text {-subtraction }},
$$

which is finite. With the bounce solution $\bar{\phi}$, the explicit expression of $\delta \mathcal{S}_{\overline{\mathrm{MS}}}^{\left(A_{\mu}, \varphi\right)}$ is given by

$$
\begin{aligned}
\delta \mathcal{S}_{\overline{\mathrm{MS}}}^{\left(A_{\mu}, \varphi\right)}= & {\left[g^{2} \delta \bar{\phi}^{2}\right]_{\mathrm{FT}}(0) \mathcal{I}_{1}\left(g^{2} v^{2}\right) } \\
& -\frac{1}{2} \int \frac{d^{4} k}{(2 \pi)^{4}}\left[g^{2} \delta \bar{\phi}^{2}\right]_{\mathrm{FT}}(-k)\left[g^{2} \delta \bar{\phi}^{2}\right]_{\mathrm{FT}}(k) \mathcal{I}_{2}\left(k^{2} ; g^{2} v^{2}, g^{2} v^{2}\right) \\
& +\frac{1}{2}[\delta \Omega]_{\mathrm{FT}}(0) \mathcal{I}_{1}(\widehat{\Omega}) \\
& -\frac{1}{4} \int \frac{d^{4} k}{(2 \pi)^{4}}[\delta \Omega]_{\mathrm{FT}}(-k)[\delta \Omega]_{\mathrm{FT}}(k) \mathcal{I}_{2}\left(k^{2} ; \widehat{\Omega}, \widehat{\Omega}\right) \\
& -2 \int \frac{d^{4} k}{(2 \pi)^{2}}[g \bar{\phi}]_{\mathrm{FT}}(-k)[g \bar{\phi}]_{\mathrm{FT}}(k) k^{2} \mathcal{I}_{2}\left(k^{2} ; g^{2} v^{2}, \widehat{\Omega}\right),
\end{aligned}
$$

where $[F]_{\mathrm{FT}}(k)$ is the Fourier transformation of the function $F(x)$ :

$$
[F]_{\mathrm{FT}}(k) \equiv \int d^{4} x e^{i k x} F(x),
$$

and the loop functions $\mathcal{I}_{1}$ and $\mathcal{I}_{2}$ are given by

$$
\begin{aligned}
16 \pi^{2} \mathcal{I}_{1}\left(m^{2}\right) \equiv & m^{2}\left(\ln \frac{m^{2}}{\mu^{2}}-1\right) \\
16 \pi^{2} \mathcal{I}_{2}\left(k^{2} ; m_{1}^{2}, m_{2}^{2}\right) \equiv & -\frac{1}{2} \ln \frac{m_{1}^{2}}{\mu^{2}}-\frac{1}{2} \ln \frac{m_{2}^{2}}{\mu^{2}}+2-\frac{m_{1}^{2}-m_{2}^{2}}{2 k^{2}} \ln \frac{m_{2}^{2}}{m_{1}^{2}} \\
& -\frac{\beta\left(k^{2} ; m_{1}^{2}, m_{2}^{2}\right)}{2} \ln \frac{k^{2}+m_{1}^{2}+m_{2}^{2}+k^{2} \beta\left(k^{2} ; m_{1}^{2}, m_{2}^{2}\right)}{k^{2}+m_{1}^{2}+m_{2}^{2}-k^{2} \beta\left(k^{2} ; m_{1}^{2}, m_{2}^{2}\right)},
\end{aligned}
$$

with

$$
\beta\left(k^{2} ; m_{1}^{2}, m_{2}^{2}\right) \equiv \frac{\sqrt{\left(k^{2}\right)^{2}+2 k^{2}\left(m_{1}^{2}+m_{2}^{2}\right)+\left(m_{1}^{2}-m_{2}^{2}\right)^{2}}}{k^{2}},
$$

and $\mu$ being the renormalization scale.

Because the bounce configuration is $O(4)$ symmetric, we can simplify the expression of $\delta \mathcal{S}_{\overline{\mathrm{MS}}}^{\left(A_{\mu}, \varphi\right)}$. Redefining $k=\sqrt{k_{\mu} k_{\mu}}$, the Fourier transformations of spherically symmetric functions are given by

$$
[F]_{\mathrm{FT}}(k)=4 \pi^{2} \int_{0}^{\infty} d r r^{3} \frac{J_{1}(k r)}{k r} F(r)
$$


Then,

$$
\begin{aligned}
\delta \mathcal{S}_{\overline{\mathrm{MS}}}^{\left(A_{\mu}, \varphi\right)}= & {\left[g^{2} \delta \bar{\phi}^{2}\right]_{\mathrm{FT}}(0) \mathcal{I}_{1}\left(g^{2} v^{2}\right)-\frac{1}{2} \int_{0}^{\infty} \frac{k^{3} d k}{8 \pi^{2}}\left[g^{2} \delta \bar{\phi}^{2}\right]_{\mathrm{FT}}^{2}(k) \mathcal{I}_{2}\left(k^{2} ; g^{2} v^{2}, g^{2} v^{2}\right) } \\
& +\frac{1}{2}[\delta \Omega]_{\mathrm{FT}}(0) \mathcal{I}_{1}(\widehat{\Omega})-\frac{1}{4} \int_{0}^{\infty} \frac{k^{3} d k}{8 \pi^{2}}[\delta \Omega]_{\mathrm{FT}}^{2}(k) \mathcal{I}_{2}\left(k^{2} ; \widehat{\Omega}, \widehat{\Omega}\right) \\
& -2 \int_{0}^{\infty} \frac{k^{3} d k}{8 \pi^{2}}[g \bar{\phi}]_{\mathrm{FT}}^{2}(k) k^{2} \mathcal{I}_{2}\left(k^{2} ; g^{2} v^{2}, \widehat{\Omega}\right)
\end{aligned}
$$

Based on the above argument, the functional determinant is renormalized as follows:

$$
\left[\operatorname{Det} \mathcal{M}^{\left(A_{\mu}, \varphi\right)}\right]^{-1 / 2} \rightarrow e^{-\delta \mathcal{S}_{\overline{\mathrm{MS}}}^{\left(A_{\mu}, \varphi\right)}} e^{\delta \mathcal{S}_{\mathrm{div}}^{\left(A_{\mu}, \varphi\right)}}\left[\operatorname{Det} \mathcal{M}^{\left(A_{\mu}, \varphi\right)}\right]^{-1 / 2}
$$

The decay rate of the false vacuum is evaluated as $\gamma=\mathcal{A} e^{-\mathcal{B}}$, with

$$
\mathcal{A}=\frac{\mathcal{B}^{2}}{4 \pi^{2}} \mathcal{A}_{\mathrm{R}}^{(h)} \mathcal{A}_{\mathrm{R}}^{(S, L, \varphi)} \mathcal{A}_{\mathrm{R}}^{(T)} \mathcal{A}_{\mathrm{R}}^{(\bar{c}, c)} e^{-\delta \mathcal{S}_{\overline{\mathrm{MS}}}^{(\mathrm{tot})}}
$$

Here,

$$
\delta \mathcal{S}_{\overline{\mathrm{MS}}}^{(\mathrm{tot})}=\delta \mathcal{S}_{\overline{\mathrm{MS}}}^{\left(A_{\mu}, \varphi\right)}+\cdots,
$$

where "..." indicates the contributions from the fields other than $A_{\mu}$ and $\varphi$ (i.e., the Higgs mode, for example). In addition, the subscript " $\mathrm{R}$ " is for "renormalized" objects after subtracting the divergences. For the $S, L$, and NG modes,

$$
\mathcal{A}_{\mathrm{R}}^{(S, L, \varphi)}=e^{s_{0}^{(S, L, \varphi)}}\left[\frac{\operatorname{Det} \mathcal{M}_{0}^{(S, \varphi)}}{\operatorname{Det} \widehat{\mathcal{M}}_{0}^{(S, \varphi)}}\right]^{-1 / 2} \prod_{J=1 / 2}^{\infty} e^{s_{J}^{(S, L, \varphi)}}\left[\frac{\operatorname{Det} \mathcal{M}_{J}^{(S, L, \varphi)}}{\operatorname{Det} \widehat{\mathcal{M}}_{J}^{(S, L, \varphi)}}\right]^{-(2 J+1)^{2} / 2},
$$

while the contribution of the transverse mode is

$$
\mathcal{A}_{\mathrm{R}}^{(T)}=e^{s_{0}^{(T)}} \prod_{J=1 / 2}^{\infty} e^{s_{J}^{(T)}}\left[\frac{\operatorname{Det} \mathcal{M}_{J}^{(T)}}{\operatorname{Det} \widehat{\mathcal{M}}_{J}^{(T)}}\right]^{-(2 J+1)^{2}} .
$$

The expressions for the functional determinants for the case of $v \neq 0(v=0)$ are given in eqs. (4.26) and (4.27) (eqs. (5.52) and (5.53)). Furthermore, the ghost contribution is

$$
\mathcal{A}_{\mathrm{R}}^{(\bar{c}, c)}=1 .
$$

Obviously, the decay rate is gauge invariant even after the renormalization.

\section{Conclusions and discussion}

In this paper, we have studied the false vacuum decay in gauge theory, paying particular attention to the gauge invariance of the decay rate of the false vacuum. Using the model with $\mathrm{U}(1)$ gauge symmetry, for which the scalar field responsible for the metastability of the false vacuum has non-vanishing charge, we have shown that the decay rate of the false 
vacuum is indeed gauge invariant at least at the one-loop level. We have adopted the gauge fixing function of the form $\mathcal{F}=\partial_{\mu} A_{\mu}$. We emphasize that such a choice of gauge fixing function is advantageous when the gauge symmetry is preserved in the false vacuum. This is because the zero-mode fluctuation in association with the internal symmetry can be successfully integrated out. Such an integration was hardly performed with the $R_{\xi}$-like gauge fixing function. We have also discussed a procedure to perform the renormalization to remove divergences, and have shown that the decay rate of the false vacuum is gauge independent even after the renormalization.

Our main results are summarized at the end of section 6 (see eqs. (6.22)-(6.26)). The decay rate of the false vacuum is related to the asymptotic values of the solutions of the second-order differential equations which are gauge independent (i.e., $f^{(\eta)}, f^{(T)}$, and so on); for a given scalar potential with a false vacuum, the second-order differential equations can be solved numerically once the bounce configuration is determined. Our results simplify the numerical calculation of the decay rate because we only have to study evolution of the gauge-invariant functions which do not mix with the other functions. In a brute-force calculation, on the contrary, one should solve simultaneous differential equations containing unphysical modes, which makes the numerical calculation unstable.

Our results would have various phenomenological applications because false vacuum decay and phase transition are important subjects in particle physics and cosmology. For example, with the measurements of the Higgs mass at the LHC experiment [16] as well as those of top mass [17], it has been realized that the standard-model Higgs potential becomes unstable when the Higgs amplitude becomes extremely large $[8,18-23]{ }^{4}$ In various models of physics beyond the standard model, the metastable vacua also show up. Supersymmetry is one of the important examples because there may exist color and/or charge breaking minima of the scalar potential [24-33].

\section{Acknowledgments}

The authors thank P. Cox for his careful reading of the manuscript. This work was supported by the Grant-in-Aid for Scientific Research on Scientific Research B (No. 16H03991 [ME and MMN] and No. 26287039 [MMN]), Scientific Research C (No. 26400239 [TM]), Young Scientists B (No. 16K17681 [ME]) and Innovative Areas (No. 16H06490 [TM] and 16H06492 [MMN]), and by World Premier International Research Center Initiative (WPI Initiative), MEXT, Japan.

\section{A Functional determinant}

In this appendix, we study eq. (2.35). A mathematical proof of a formula similar to eq. (2.35) has been provided in $[14,15]$. Because the set up adopted in $[14,15]$ is different from ours, we give a proof for the functional determinants of $\mathcal{M}_{J}^{(S, L, \varphi)}$ and $\mathcal{M}_{0}^{(S, \varphi)}$. For the

\footnotetext{
${ }^{4}$ In the case of the Higgs potential of the standard model, which is dominated by the quartic term when calculating the decay rate of the false vacuum, the asymptotic behavior of the bounce at $r \rightarrow \infty$ is different from that adopted in the present analysis. Such a case will be studied elsewhere.
} 
fluctuation operators of the transverse mode, Higgs mode, and FP ghosts, similar argument holds and we can use eq. (2.35). In this appendix, $\mathcal{M}(\widehat{\mathcal{M}})$ denotes $\mathcal{M}_{J}^{(S, L, \varphi)}$ or $\mathcal{M}_{0}^{(S, \varphi)}$ $\left(\widehat{\mathcal{M}}_{J}^{(S, L, \varphi)}\right.$ or $\left.\widehat{\mathcal{M}}_{0}^{(S, \varphi)}\right)$.

In order to explicitly impose the boundary condition to the eigenfunctions, we first consider the functional determinant for the eigenfunctions defined in the finite interval $0<r \leq R$; at the end of the calculation, we take $R \rightarrow \infty$. Let us denote the $n$-th eigenvalues of $\mathcal{M}$ and $\widehat{\mathcal{M}}$ as $\lambda_{n}$ and $\widehat{\lambda}_{n}$, respectively. Then, the corresponding eigenfunctions, denoted as $\psi_{n}$ and $\widehat{\psi}_{n}$, respectively, satisfy

$$
\begin{aligned}
& \mathcal{M} \psi_{n}=\lambda_{n} \psi_{n}, \\
& \widehat{\mathcal{M}} \widehat{\psi}_{n}=\widehat{\lambda}_{n} \widehat{\psi}_{n},
\end{aligned}
$$

with

$$
\psi_{n}(R)=\widehat{\psi}_{n}(R)=0
$$

Notice that we are interested in the functional determinant of Hermitian operators, and hence $\lambda_{n}$ and $\hat{\lambda}_{n}$ are real.

We start with introducing the $\zeta$ functions:

$$
\begin{aligned}
\zeta_{\mathcal{M}}(s) & \equiv \sum_{n} \lambda_{n}^{-s}, \\
\zeta_{\widehat{\mathcal{M}}}(s) & \equiv \sum_{n} \widehat{\lambda}_{n}^{-s} .
\end{aligned}
$$

Then, the ratio of the functional determinants is defined as

$$
\frac{\operatorname{Det} \mathcal{M}}{\operatorname{Det} \widehat{\mathcal{M}}}=e^{-\left[\zeta_{\mathcal{M}}^{\prime}(0)-\zeta_{\widehat{\mathcal{M}}}^{\prime}(0)\right]}
$$

For the calculation of the $\zeta$ functions defined above, we introduce the function $u(r ; \lambda)$, which satisfies

$$
\mathcal{M} u(r ; \lambda)=\lambda u(r ; \lambda)
$$

where $\lambda$ is a complex constant, and $u(r \rightarrow 0 ; \lambda)$ is required to be finite.

We first show that there are three (two) independent choices of $u(r ; \lambda)$ for $J \neq 0$ $(J=0)$. To see this, we expand $u(r ; \lambda)$ as

$$
u(r ; \lambda)=r^{\nu} \sum_{p=0}^{\infty} c_{p} r^{p},
$$

where $\nu$ is a non-negative constant because of the regularity at the origin, and $c_{p}$ are constant 3 -component (2-component) objects for $J \neq 0(J=0)$ satisfying $c_{0} \neq 0$. In order for $u(r ; \lambda)$ to obey eq. (A.7), the following relation should hold:

$$
\mathcal{M}^{\star} r^{\nu} c_{0}=0
$$


where $\mathcal{M}^{\star}$ is obtained from $\mathcal{M}$ by taking $\bar{\phi} \rightarrow 0, \bar{\phi}^{\prime} \rightarrow 0$, and $\Delta_{0} \bar{\phi} \rightarrow 0$; it gives terms with the lowest power in $r$ (i.e., terms of $O\left(r^{\nu-2}\right)$ ) in eq. (A.7). The above equation has solutions if $\operatorname{det}\left[r^{2-\nu} \mathcal{M}^{\star} r^{\nu}\right]=0$. (Notice that $r^{2-\nu} \mathcal{M}^{\star} r^{\nu}$ is a constant $3 \times 3(2 \times 2)$ matrix for $J \neq 0(J=0)$.) It gives the following values of $\nu$ :

$$
\nu=\left\{\begin{array}{ll}
2 J-1,2 J, 2 J+1 & : J \neq 0 \\
0,1 & : J=0
\end{array} .\right.
$$

We can repeat the above argument for $\widehat{\mathcal{M}}$ to define the function $\widehat{u}(r ; \lambda)$, which satisfies

$$
\widehat{\mathcal{M}} \widehat{u}(r ; \lambda)=\lambda \widehat{u}(r ; \lambda) .
$$

We can see that $u(r \rightarrow 0 ; \lambda)$ and $\widehat{u}(r \rightarrow 0 ; \lambda)$ have the same power-law behavior at $r \rightarrow 0$. We choose the boundary conditions as follows: for $J \neq 0$,

$$
\begin{aligned}
& u_{1}(r \rightarrow 0 ; \lambda) \simeq \widehat{u}_{1}(r \rightarrow 0 ; \lambda) \simeq\left(\begin{array}{c}
2 J r^{2 J-1} \\
L r^{2 J-1} \\
0
\end{array}\right), \\
& u_{2}(r \rightarrow 0 ; \lambda) \simeq \widehat{u}_{2}(r \rightarrow 0 ; \lambda) \simeq\left(\begin{array}{c}
0 \\
0 \\
r^{2 J}
\end{array}\right) \\
& u_{3}(r \rightarrow 0 ; \lambda) \simeq \widehat{u}_{3}(r \rightarrow 0 ; \lambda) \simeq\left(\begin{array}{c}
\frac{(J+1) \xi-J}{2 L^{2}} r^{2 J+1} \\
\frac{(J+1) \xi-(J+2)}{4 L(J+1)} r^{2 J+1} \\
0
\end{array}\right),
\end{aligned}
$$

and for $J=0$,

$$
\begin{aligned}
& u_{1}(r \rightarrow 0 ; \lambda) \simeq \widehat{u}_{1}(r \rightarrow 0 ; \lambda) \simeq\left(\begin{array}{l}
0 \\
1
\end{array}\right), \\
& u_{2}(r \rightarrow 0 ; \lambda) \simeq \widehat{u}_{2}(r \rightarrow 0 ; \lambda) \simeq\left(\begin{array}{l}
r \\
0
\end{array}\right) .
\end{aligned}
$$

Now we express the $\zeta$ functions using the functions $u_{I}$ and $\widehat{u}_{I}$. To make our argument explicit, we concentrate on the case of $J \neq 0$; similar argument holds for the case of $J=0$. For the calculation the $\zeta$ functions, we use the following relation:

$$
\begin{aligned}
& \operatorname{det}\left(u_{1}\left(r=R ; \lambda=\lambda_{n}\right) u_{2}\left(r=R ; \lambda=\lambda_{n}\right) u_{3}\left(r=R ; \lambda=\lambda_{n}\right)\right)=0, \\
& \operatorname{det}\left(\widehat{u}_{1}\left(r=R ; \lambda=\widehat{\lambda}_{n}\right) \widehat{u}_{2}\left(r=R ; \lambda=\widehat{\lambda}_{n}\right) \widehat{u}_{3}\left(r=R ; \lambda=\widehat{\lambda}_{n}\right)\right)=0,
\end{aligned}
$$

which are based on the boundary conditions on the eigenfunctions of the differential operators (see eq. (A.3)). Thus, the logarithmic derivatives of above determinants with respect to $\lambda$ have simple poles with unit residue at the eigenvalues of corresponding fluctuation operators, and hence we can express the $\zeta$ functions as $[14,15]$

$$
\zeta_{\mathcal{M}}(s)-\zeta_{\widehat{\mathcal{M}}}(s)=\frac{1}{2 \pi i} \int_{C_{0}} d \lambda \lambda^{-s} \frac{d}{d \lambda} \ln \frac{\operatorname{det}\left(u_{1}(R ; \lambda) u_{2}(R ; \lambda) u_{3}(R ; \lambda)\right)}{\operatorname{det}\left(\widehat{u}_{1}(R ; \lambda) \widehat{u}_{2}(R ; \lambda) \widehat{u}_{3}(R ; \lambda)\right)}
$$



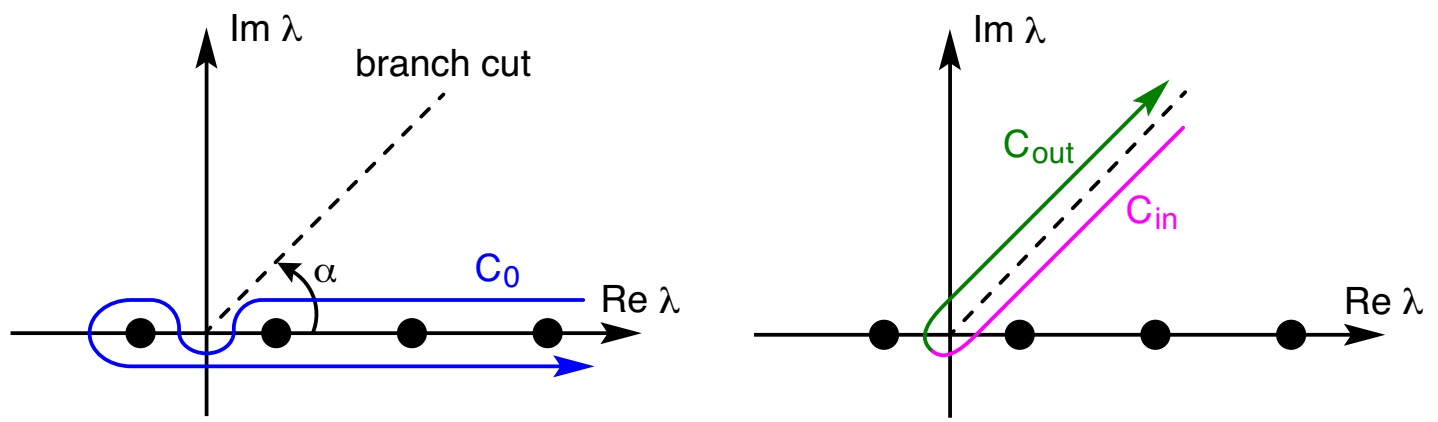

Figure 1. The contours $C_{0}, C_{\text {in }}$, and $C_{\text {out }}$ on the complex $\lambda$ plane. The blobs on the real axis indicate the eigenvalues of the fluctuation operators. The dotted line is the branch cut of $\lambda^{-s}$.

where $C_{0}$ is a contour along the real axis, surrounding all eigenvalues in counterclockwise direction (see figure 1). The contour $C_{0}$ avoids the branch cut of $\lambda^{-s}$, which is defined to be a straight line starting from the origin with the angle $\alpha$ to the real axis.

The next task is to deform the contour to enclose the branch cut of $\lambda^{-s}$. To do so, information about the behavior of the integrand at $|\lambda| \rightarrow \infty$ is necessary. We can use the fact that $u_{I}(r ; \lambda)$ should satisfy

$$
u_{I}(r ; \lambda)=u_{I}^{\star}(r)+\int^{r} d r_{1} r_{1}^{3} G\left(r, r_{1} ; \lambda\right) \delta \mathcal{M}\left(r_{1}\right) u_{I}\left(r_{1} ; \lambda\right),
$$

where $\delta \mathcal{M} \equiv \mathcal{M}-\mathcal{M}^{\star}$. Here, the function $G$ is given by

$$
\begin{aligned}
G\left(r_{1}, r_{2} ; \lambda\right) \equiv & \frac{\pi \lambda \xi^{2}}{2}\left[v_{1}\left(r_{1} ; \lambda\right) w_{1}^{T}\left(r_{2} ; \lambda\right)-w_{1}\left(r_{1} ; \lambda\right) v_{1}^{T}\left(r_{2} ; \lambda\right)\right] \\
& +\frac{\pi \lambda}{2}\left[v_{2}\left(r_{1} ; \lambda\right) w_{2}^{T}\left(r_{2} ; \lambda\right)-w_{2}\left(r_{1} ; \lambda\right) v_{2}^{T}\left(r_{2} ; \lambda\right)\right] \\
& +\frac{\pi \lambda}{2}\left[v_{3}\left(r_{1} ; \lambda\right) w_{3}^{T}\left(r_{2} ; \lambda\right)-w_{3}\left(r_{1} ; \lambda\right) v_{3}^{T}\left(r_{2} ; \lambda\right)\right],
\end{aligned}
$$

where

$$
\begin{aligned}
& v_{1}(r ; \lambda) \equiv\left(\begin{array}{c}
\partial_{r} \\
\frac{L}{r} \\
0
\end{array}\right) \frac{J_{2 J+1}(\sqrt{\lambda \xi} r)}{\lambda \xi r}, \\
& v_{2}(r ; \lambda) \equiv\left(\begin{array}{c}
\frac{L}{r} \\
\frac{1}{r^{2}} \partial_{r} r^{2} \\
0
\end{array}\right) \frac{J_{2 J+1}(\sqrt{\lambda} r)}{\lambda r}, \\
& v_{3}(r ; \lambda) \equiv\left(\begin{array}{c}
0 \\
0 \\
1
\end{array}\right) \frac{J_{2 J+1}(\sqrt{\lambda} r)}{\sqrt{\lambda} r},
\end{aligned}
$$

with $J_{2 J+1}$ being the Bessel function of the first kind, and $w_{I}$ are obtained from $v_{I}$ by replacing $J_{2 J+1} \rightarrow N_{2 J+1}$ (with $N_{2 J+1}$ being the Bessel function of the second kind). 
Furthermore, $u_{I}^{\star}$ are solutions of the following differential equation:

$$
\left(\mathcal{M}^{\star}-\lambda\right) u_{I}^{\star}=0
$$

which are regular at $r \rightarrow 0$. Requiring the boundary conditions given in eq. (A.12), (A.13), and (A.14) for $u_{1}^{\star}, u_{2}^{\star}$, and $u_{3}^{\star}$, respectively,

$$
\begin{aligned}
& u_{1}^{\star}(r)=4 \Gamma(2 J+2)\left(\frac{\sqrt{\xi \lambda}}{2}\right)^{-(2 J-1)} v_{1}(r ; \lambda), \\
& u_{2}^{\star}(r)=2 \Gamma(2 J+2)\left(\frac{\sqrt{\lambda}}{2}\right)^{-2 J} v_{3}(r ; \lambda), \\
& u_{3}^{\star}(r)=\frac{4 \Gamma(2 J+2)}{\lambda L}\left(\frac{\sqrt{\lambda}}{2}\right)^{-(2 J-1)} v_{2}(r ; \lambda)-\frac{2 \Gamma(2 J+2)}{\lambda J}\left(\frac{\sqrt{\xi \lambda}}{2}\right)^{-(2 J-1)} v_{1}(r ; \lambda) .
\end{aligned}
$$

With large enough $\lambda$, we can see that the second term in the right-hand side of eq. (A.20) is at most $O\left(|\lambda|^{-1 / 2}\right)$ compared to the first term. This can be understood by rewriting eq. (A.20) with a new dimensionless variable $\rho \equiv \sqrt{|\lambda|} r$; with such a new variable, $\delta \mathcal{M}$ can be treated as a perturbation and we can expand the solution with respect to $\bar{\phi}_{\max } / \sqrt{|\lambda|}$ where $\bar{\phi}_{\max }$ is the scale of the maximal amplitude of the bounce configuration. Thus, we expect that

$$
u_{I}(r ; \lambda \rightarrow \infty) \simeq u_{I}^{\star}(r)+O\left(\lambda^{-1 / 2}\right) .
$$

A similar analysis applies to $\widehat{u}_{I}$, which results in

$$
\widehat{u}_{I}(r ; \lambda \rightarrow \infty) \simeq u_{I}^{\star}(r)+O\left(\lambda^{-1 / 2}\right)
$$

Thus, $u_{I}$ and $\widehat{u}_{I}$ have almost the same functional form when $\lambda \rightarrow \infty$ and, for $\operatorname{Im} \lambda \neq 0$,

$$
\left.\frac{d}{d \lambda} \ln \frac{\operatorname{det}\left(u_{1}(R ; \lambda) u_{2}(R ; \lambda) u_{3}(R ; \lambda)\right)}{\operatorname{det}\left(\widehat{u}_{1}(R ; \lambda) \widehat{u}_{2}(R ; \lambda) \widehat{u}_{3}(R ; \lambda)\right)}\right|_{\lambda \rightarrow \infty} \sim O\left(\lambda^{-3 / 2}\right)
$$

This implies that, when Res $>-\frac{1}{2}$, the integration at $\lambda \rightarrow \infty$ does not contribute. ${ }^{5}$

Based on the above consideration, we can replace $\int_{C_{0}} \rightarrow \int_{C_{\text {in }}+C_{\text {out }}}$, where the contours $C_{\text {in }}$ and $C_{\text {out }}$ are those along the branch cut incoming to and outgoing from the origin (see figure 1). Using the fact that the functions $u_{I}$ and $\widehat{u}_{I}$ are continuous at the branch cut of $\lambda^{-s}$,

$$
\zeta_{\mathcal{M}}(s)-\zeta_{\widehat{\mathcal{M}}}(s)=e^{i s(\pi-\alpha)} \frac{\sin (\pi s)}{\pi} \int_{0}^{\infty} d \lambda \lambda^{-s} \frac{d}{d \lambda} \ln \frac{\operatorname{det}\left(u_{1}\left(R ; e^{i \alpha} \lambda\right) u_{2}\left(R ; e^{i \alpha} \lambda\right) u_{3}\left(R ; e^{i \alpha} \lambda\right)\right)}{\operatorname{det}\left(\widehat{u}_{1}\left(R ; e^{i \alpha} \lambda\right) \widehat{u}_{2}\left(R ; e^{i \alpha} \lambda\right) \widehat{u}_{3}\left(R ; e^{i \alpha} \lambda\right)\right)}
$$

\footnotetext{
${ }^{5}$ For the calculation of $\zeta_{\mathcal{M}}(s)-\zeta_{\widehat{\mathcal{M}}}(s)$, convergence of the integration around $\lambda \sim 0$ requires $\operatorname{Re} s<1$.
} 
Combining the above result with eq. (A.6), and taking $R \rightarrow \infty$, we come to the most important formula in this appendix: ${ }^{6}$

$$
\frac{\operatorname{Det} \mathcal{M}}{\operatorname{Det} \widehat{\mathcal{M}}}=\frac{\operatorname{det}\left(u_{1}\left(r_{\infty} ; \lambda=0\right) u_{2}\left(r_{\infty} ; \lambda=0\right) u_{3}\left(r_{\infty} ; \lambda=0\right)\right)}{\operatorname{det}\left(\widehat{u}_{1}\left(r_{\infty} ; \lambda=0\right) \widehat{u}_{2}\left(r_{\infty} ; \lambda=0\right) \widehat{u}_{3}\left(r_{\infty} ; \lambda=0\right)\right)} .
$$

Notice that, for the convergence of the above quantity, $\mathcal{M}$ and $\widehat{\mathcal{M}}$ should have the same asymptotic behavior at $r \rightarrow \infty$, which holds for the case of our interest. In the discussion given in sections 4 and 5, the initial conditions of the solutions of eqs. (A.7) and (A.11) are taken to be different. (However, notice that we take three independent solutions for $\mathcal{M}_{J}^{(S, L, \varphi)}$ and $\widehat{\mathcal{M}}_{J}^{(S, L, \varphi)}$, which are linear combinations of $u_{I}$ and $\widehat{u}_{I}$, respectively.) Then, we should use eq. (2.35).

\section{B Solutions of inhomogeneous differential equation}

In this appendix, we discuss the asymptotic behavior of the inhomogeneous differential equation of the following form:

$$
\left(\Delta_{J}-m^{2}\right) F(r)=S(r),
$$

where $m$ is a constant, and the source term $S$ behaves as

$$
S(r \rightarrow \infty) \simeq s_{0} r^{-p} e^{\lambda r}
$$

Using the modified Bessel functions, $I_{2 J+1}$ and $K_{2 J+1}$, the solution of the above equation is given by

$$
\begin{aligned}
F(r)= & c_{I} \frac{I_{2 J+1}(m r)}{r}+c_{K} \frac{K_{2 J+1}(m r)}{r} \\
& +\frac{1}{r}\left[I_{2 J+1}(m r) \int^{r} d r^{\prime} r^{\prime 2} K_{2 J+1}\left(m r^{\prime}\right) S\left(r^{\prime}\right)-K_{2 J+1}(m r) \int^{r} d r^{\prime} r^{\prime 2} I_{2 J+1}\left(m r^{\prime}\right) S\left(r^{\prime}\right)\right],
\end{aligned}
$$

where $c_{I}$ and $c_{K}$ are constants.

Using the properties of the modified Bessel functions, i.e., $I_{2 J+1}(z \rightarrow \infty) \simeq(2 \pi z)^{-1 / 2} e^{z}$ and $K_{2 J+1}(z \rightarrow \infty) \simeq(\pi / 2 z)^{1 / 2} e^{-z}$, the asymptotic behavior of the function $F(r)$ with the source term given in eq. (B.2) can be expressed as ${ }^{7}$

$$
F(r \rightarrow \infty) \simeq c_{I} \frac{I_{2 J+1}(m r)}{r}+c_{K} \frac{K_{2 J+1}(m r)}{r}+\frac{s_{0}}{\lambda^{2}-m^{2}} r^{-p} e^{\lambda r} .
$$

\footnotetext{
${ }^{6}$ Instead of eq. (A.3), we may take an alternative boundary condition: $\psi_{n}^{\prime}(R)=\widehat{\psi}_{n}^{\prime}(R)=0$. A similar argument holds for this boundary condition; the result is given by eq. (A.33) with replacing $u_{I} \rightarrow u_{I}^{\prime}$ and $\widehat{u}_{I} \rightarrow \widehat{u}_{I}^{\prime}$.

${ }^{7}$ Notice that, with the asymptotic behaviors of the modified Bessel functions, the integrations in eq. (B.3) can be expressed by using the incomplete gamma function as

$$
\begin{aligned}
\int_{r_{0}}^{r} d r^{\prime} r^{\prime 2} I_{2 J+1}\left(m r^{\prime}\right) S\left(r^{\prime}\right) & \simeq \frac{1}{\sqrt{2 \pi m}}(-m-\lambda)^{q}\left[\Gamma\left(q,-(m+\lambda) r_{0}\right)-\Gamma(q,-(m+\lambda) r)\right], \\
\int_{r_{0}}^{r} d r^{\prime} r^{\prime 2} K_{2 J+1}\left(m r^{\prime}\right) S\left(r^{\prime}\right) & \simeq \sqrt{\frac{\pi}{2 m}}(m-\lambda)^{q}\left[\Gamma\left(q,(m+\lambda) r_{0}\right)-\Gamma(q,(m+\lambda) r)\right],
\end{aligned}
$$

with $q=(2 p-5) / 2$. (Here, $m r_{0} \gg 1$ and $m r \gg 1$ are assumed.) In addition, in deriving eq. (B.4), we also use $\Gamma(q, z \rightarrow \pm \infty) \simeq z^{q-1} e^{-z}$.
} 
In the above expressions, the arbitrariness of the lower boundaries of the integrations are absorbed into the constants $c_{I}$ and $c_{K}$.

\section{Functional determinant with small perturbations}

In this appendix, we outline the prescription to calculate $\delta \mathcal{S}_{\operatorname{div}}^{(S, L, \varphi)}$ and $\delta \mathcal{S}_{\text {div }}^{(T)}$ given in eqs. (6.8) and (6.9), respectively. We expand the functional determinants with respect to small perturbations, and calculate the functional determinant up to a finite order of the perturbation.

The second term of $\delta \mathcal{S}_{\text {div }}^{(S, L, \varphi)}$ as well as $\delta \mathcal{S}_{\text {div }}^{(T)}$ are described by the following quantity with $N=2$ :

$$
\ell^{[N]} \equiv\left[\ln \frac{\operatorname{Det}\left(-\partial^{2}+g^{2} v^{2}+g^{2} \delta \bar{\phi}^{2}\right)}{\operatorname{Det}\left(-\partial^{2}+g^{2} v^{2}\right)}\right]_{\left(g^{2} \delta \bar{\phi}^{2}\right)^{N}},
$$

where, as introduced in section $6,[\cdots]_{\mathcal{P}^{N}}$ indicates that the quantity in the square bracket is evaluated up to $O\left(\mathcal{P}^{N}\right)$. Using the angular-momentum decomposition, and also using eq. (2.35), the ratio of the functional determinants in eq. (C.1) is given by

$$
e^{\ell[N=\infty]}=\prod_{J}\left[\frac{f_{J}\left(r_{\infty}\right)}{\widehat{f}_{J}\left(r_{\infty}\right)}\right]^{(2 J+1)^{2}},
$$

where the functions $f_{J}$ and $\widehat{f}_{J}$ satisfy

$$
\begin{aligned}
\left(\Delta_{J}-g^{2} v^{2}-g^{2} \delta \bar{\phi}^{2}\right) f_{J} & =0, \\
\left(\Delta_{J}-g^{2} v^{2}\right) \widehat{f}_{J} & =0,
\end{aligned}
$$

with $f_{J}(r \rightarrow 0) \simeq \widehat{f}_{J}(r \rightarrow 0) \simeq r^{2 J}$.

Treating $\delta \bar{\phi}^{2}$ as a perturbation, we expand the function $f_{J}$ as

$$
f_{J}(r)=\sum_{n=0}^{\infty} f_{J}^{(n)}(r)
$$

where $f_{J}^{(n)}$ is obtained by iteratively solving

$$
\left(\Delta_{J}-g^{2} v^{2}\right) f_{J}^{(n)}=g^{2} \delta \bar{\phi}^{2} f_{J}^{(n-1)},
$$

with $f_{J}^{(0)}=\widehat{f}_{J}$ and $f_{J}^{(n \neq 0)}(r \rightarrow 0) \sim O\left(r^{2 J+1}\right)$.

In order for the calculation of the counter terms for each angular momentum, we decompose $\ell^{[N]}$ as

$$
\ell^{[N]}=\sum_{J=0}^{\infty} \ell_{J}^{[N]}
$$

Then,

$$
\ell_{J}^{[N=\infty]}=(2 J+1)^{2} \ln \left[\sum_{n=0}^{\infty} t^{n}\left(\frac{f_{J}^{(n)}\left(r_{\infty}\right)}{\widehat{f}_{J}\left(r_{\infty}\right)}\right)\right]_{t \rightarrow 1}
$$


For the calculation of $\ell_{J}^{[N]}$, we expand the right-hand side of the above equation with respect to $t$, neglect terms of $O\left(t^{p}\right)$ with $p \geq N+1$, and take $t \rightarrow 1$. In particular,

$$
\ell_{J}^{[N=2]}=(2 J+1)^{2}\left[\left(\frac{f_{J}^{(1)}\left(r_{\infty}\right)}{\widehat{f}_{J}\left(r_{\infty}\right)}\right)+\left(\frac{f_{J}^{(2)}\left(r_{\infty}\right)}{\widehat{f}_{J}\left(r_{\infty}\right)}\right)-\frac{1}{2}\left(\frac{f_{J}^{(1)}\left(r_{\infty}\right)}{\widehat{f}_{J}\left(r_{\infty}\right)}\right)^{2}\right] .
$$

Eq. (6.8) contains functional determinants of the fluctuation operators in the matrix form. Even in such a case, we can expand the functional determinant of our interest with respect to perturbations. For the calculation of the first term of the right-hand side of eq. (6.8), we introduce $3 \times 3$ functions $\mathbf{F}_{J}(r)$ for $J>0$, which are expanded as

$$
\mathbf{F}_{J}(r)=\sum_{n=0}^{\infty} \mathbf{F}_{J}^{(n)}(r)
$$

They obey the following differential equations:

$$
\left(\begin{array}{ccc}
\Delta_{J}-\frac{3}{r^{2}}-g^{2} v^{2} & \frac{2 L}{r^{2}} & 0 \\
\frac{2 L}{r^{2}} & \Delta_{J}+\frac{1}{r^{2}}-g^{2} v^{2} & 0 \\
0 & 0 & \Delta_{J}-\widehat{\Omega}
\end{array}\right) \mathbf{F}_{J}^{(n)}=\left(\begin{array}{ccc}
g^{2} \delta \bar{\phi}^{2} & 0 & 2 g \bar{\phi}^{\prime} \\
0 & g^{2} \delta \bar{\phi}^{2} & 0 \\
2 g \bar{\phi}^{\prime} & 0 & \delta \Omega
\end{array}\right) \mathbf{F}_{J}^{(n-1)}
$$

for $n \geq 1$, and

$$
\mathbf{F}_{J}^{(0)}(r \rightarrow 0) \simeq\left(\begin{array}{ccc}
2 J r^{2 J-1} & -L r^{2 J+1} & 0 \\
L r^{2 J-1} & 2 J r^{2 J+1} & 0 \\
0 & 0 & r^{2 J}
\end{array}\right)
$$

For $J=0, \mathbf{F}_{J=0}$ is a $2 \times 2$ object; it satisfies

$$
\left(\begin{array}{cc}
\Delta_{0}-\frac{3}{r^{2}}-g^{2} v^{2} & 0 \\
0 & \Delta_{0}-\widehat{\Omega}
\end{array}\right) \mathbf{F}_{0}^{(n)}=\left(\begin{array}{cc}
g^{2} \delta \bar{\phi}^{2} & 2 g \bar{\phi}^{\prime} \\
2 g \bar{\phi}^{\prime} & \delta \Omega
\end{array}\right) \mathbf{F}_{0}^{(n-1)},
$$

with

$$
\mathbf{F}_{0}^{(0)}(r \rightarrow 0) \simeq\left(\begin{array}{ll}
r & 0 \\
0 & 1
\end{array}\right)
$$

With the functions $\mathbf{F}_{J}$, we define

$$
\begin{aligned}
L_{J}^{[N=2]} \equiv & (2 J+1)^{2} \operatorname{tr}\left[\widehat{\mathbf{F}}_{J}^{-1}\left(r_{\infty}\right) \mathbf{F}_{J}^{(1)}\left(r_{\infty}\right)+\widehat{\mathbf{F}}_{J}^{-1}\left(r_{\infty}\right) \mathbf{F}_{J}^{(2)}\left(r_{\infty}\right)\right] \\
& -\frac{(2 J+1)^{2}}{2} \operatorname{tr}\left[\widehat{\mathbf{F}}_{J}^{-1}\left(r_{\infty}\right) \mathbf{F}_{J}^{(1)}\left(r_{\infty}\right) \widehat{\mathbf{F}}_{J}^{-1}\left(r_{\infty}\right) \mathbf{F}_{J}^{(1)}\left(r_{\infty}\right)\right]
\end{aligned}
$$

where $\widehat{\mathbf{F}}_{J} \equiv \mathbf{F}_{J}^{(0)}$. Then, the first term of eq. (6.8) is expressed as

$$
\frac{1}{2}\left[\frac{\operatorname{Det}\left(\widehat{\mathcal{M}}_{R_{\xi=1}}^{\left(A_{\mu}, \varphi\right)}+\delta \mathcal{M}\right)}{\operatorname{Det} \widehat{\mathcal{M}}_{R_{\xi=1}}^{\left(A_{\mu}, \varphi\right)}}\right]_{(\delta \mathcal{M})^{2}}=\frac{1}{2} \sum_{J=0}^{\infty} L_{J}^{[N=2]}+\sum_{J=1 / 2}^{\infty} \ell_{J}^{[N=2]} .
$$


In summary, the divergent parts given in eqs. (6.8) and (6.9) are given by

$$
\begin{aligned}
\delta \mathcal{S}_{\operatorname{div}}^{(S, L, \varphi)} & =\frac{1}{2} \sum_{J=0}^{\infty} L_{J}^{[N=2]}-\sum_{J=0}^{\infty} \ell_{J}^{[N=2]}-\ell_{0}^{[N=2]} \\
\delta \mathcal{S}_{\operatorname{div}}^{(T)} & =\sum_{J=0}^{\infty} \ell_{J}^{[N=2]} .
\end{aligned}
$$

Comparing the above equations with eq. (6.12) or (6.13), we can obtain the counter terms for each angular momentum, $s_{J}^{(S, L, \varphi)}$ and $s_{J}^{(T)}$.

Open Access. This article is distributed under the terms of the Creative Commons Attribution License (CC-BY 4.0), which permits any use, distribution and reproduction in any medium, provided the original author(s) and source are credited.

\section{References}

[1] S.R. Coleman, The Fate of the False Vacuum. 1. Semiclassical Theory, Phys. Rev. D 15 (1977) 2929 [Erratum ibid. D 16 (1977) 1248] [INSPIRE].

[2] C.G. Callan Jr. and S.R. Coleman, The Fate of the False Vacuum. 2. First Quantum Corrections, Phys. Rev. D 16 (1977) 1762 [InSPIRE].

[3] S. Coleman, The uses of instantons, in Aspects of Symmetry, Cambridge University Press (1985), pg. 265.

[4] N.K. Nielsen, On the Gauge Dependence of Spontaneous Symmetry Breaking in Gauge Theories, Nucl. Phys. B 101 (1975) 173 [INSPIRE].

[5] R. Fukuda and T. Kugo, Gauge Invariance in the Effective Action and Potential, Phys. Rev. D 13 (1976) 3469 [inSPIRE].

[6] M. Endo, T. Moroi, M.M. Nojiri and Y. Shoji, On the Gauge Invariance of the Decay Rate of False Vacuum, Phys. Lett. B 771 (2017) 281 [arXiv:1703.09304] [INSPIRE].

[7] J. Baacke and K. Heitmann, Gauge invariance of the one loop effective action of the Higgs field in the SU(2) Higgs model, Phys. Rev. D 60 (1999) 105037 [hep-th/9905201] [INSPIRE].

[8] G. Isidori, G. Ridolfi and A. Strumia, On the metastability of the standard model vacuum, Nucl. Phys. B 609 (2001) 387 [hep-ph/0104016] [InSPIRE].

[9] L.P. Alexander and A. Pilaftsis, The One-Loop Effective Potential in Non-Linear Gauges, J. Phys. G 36 (2009) 045006 [arXiv: 0809.1580] [InSPIRE].

[10] A. Kusenko, K.-M. Lee and E.J. Weinberg, Vacuum decay and internal symmetries, Phys. Rev. D 55 (1997) 4903 [hep-th/9609100] [INSPIRE].

[11] S.R. Coleman, V. Glaser and A. Martin, Action Minima Among Solutions to a Class of Euclidean Scalar Field Equations, Commun. Math. Phys. 58 (1978) 211 [inSPIRE].

[12] K. Blum, M. Honda, R. Sato, M. Takimoto and K. Tobioka, $O(N)$ Invariance of the Multi-Field Bounce, JHEP 05 (2017) 109 [Erratum ibid. 06 (2017) 060] [arXiv:1611.04570] [INSPIRE].

[13] R.F. Dashen, B. Hasslacher and A. Neveu, Nonperturbative Methods and Extended Hadron Models in Field Theory. 1. Semiclassical Functional Methods, Phys. Rev. D 10 (1974) 4114 [INSPIRE]. 
[14] K. Kirsten and A.J. McKane, Functional determinants by contour integration methods, Annals Phys. 308 (2003) 502 [math-ph/0305010] [INSPIRE].

[15] K. Kirsten and A.J. McKane, Functional determinants for general Sturm-Liouville problems, J. Phys. A 37 (2004) 4649 [math-ph/0403050] [INSPIRE].

[16] ATLAS, CMS collaborations, Combined Measurement of the Higgs Boson Mass in pp Collisions at $\sqrt{s}=7$ and 8 TeV with the ATLAS and CMS Experiments, Phys. Rev. Lett. 114 (2015) 191803 [arXiv:1503.07589] [InSPIRE].

[17] ATLAS, CDF, CMS, D0 collaborations, First combination of Tevatron and LHC measurements of the top-quark mass, arXiv:1403.4427 [INSPIRE].

[18] G. Degrassi et al., Higgs mass and vacuum stability in the Standard Model at NNLO, JHEP 08 (2012) 098 [arXiv: 1205.6497] [INSPIRE].

[19] S. Alekhin, A. Djouadi and S. Moch, The top quark and Higgs boson masses and the stability of the electroweak vacuum, Phys. Lett. B 716 (2012) 214 [arXiv:1207.0980] [InSPIRE].

[20] J.R. Espinosa et al., The cosmological Higgstory of the vacuum instability, JHEP 09 (2015) 174 [arXiv:1505.04825] [INSPIRE].

[21] A.D. Plascencia and C. Tamarit, Convexity, gauge-dependence and tunneling rates, JHEP 10 (2016) 099 [arXiv:1510.07613] [INSPIRE].

[22] Z. Lalak, M. Lewicki and P. Olszewski, Gauge fixing and renormalization scale independence of tunneling rate in Abelian Higgs model and in the standard model, Phys. Rev. D 94 (2016) 085028 [arXiv: 1605.06713] [INSPIRE].

[23] J.R. Espinosa, M. Garny, T. Konstandin and A. Riotto, Gauge-Independent Scales Related to the Standard Model Vacuum Instability, Phys. Rev. D 95 (2017) 056004 [arXiv:1608.06765] [INSPIRE].

[24] J.F. Gunion, H.E. Haber and M. Sher, Charge/Color Breaking Minima and a-Parameter Bounds in Supersymmetric Models, Nucl. Phys. B 306 (1988) 1 [INSPIRE].

[25] J.A. Casas, A. Lleyda and C. Muñoz, Strong constraints on the parameter space of the MSSM from charge and color breaking minima, Nucl. Phys. B 471 (1996) 3 [hep-ph/9507294] [INSPIRE].

[26] A. Kusenko, P. Langacker and G. Segre, Phase transitions and vacuum tunneling into charge and color breaking minima in the MSSM, Phys. Rev. D 54 (1996) 5824 [hep-ph/9602414] [INSPIRE].

[27] J. Hisano and S. Sugiyama, Charge-breaking constraints on left-right mixing of stau's, Phys. Lett. B 696 (2011) 92 [Erratum ibid. B 719 (2013) 472] [arXiv:1011.0260] [INSPIRE].

[28] J.E. Camargo-Molina, B. O'Leary, W. Porod and F. Staub, Stability of the CMSSM against sfermion VEVs, JHEP 12 (2013) 103 [arXiv:1309.7212] [INSPIRE].

[29] D. Chowdhury, R.M. Godbole, K.A. Mohan and S.K. Vempati, Charge and Color Breaking Constraints in MSSM after the Higgs Discovery at LHC, JHEP 02 (2014) 110 [arXiv: 1310.1932] [INSPIRE].

[30] N. Blinov and D.E. Morrissey, Vacuum Stability and the MSSM Higgs Mass, JHEP 03 (2014) 106 [arXiv:1310.4174] [INSPIRE]. 
[31] J.E. Camargo-Molina, B. Garbrecht, B. O'Leary, W. Porod and F. Staub, Constraining the Natural MSSM through tunneling to color-breaking vacua at zero and non-zero temperature, Phys. Lett. B 737 (2014) 156 [arXiv: 1405.7376] [INSPIRE].

[32] M. Endo, T. Moroi and M.M. Nojiri, Footprints of Supersymmetry on Higgs Decay, JHEP 04 (2015) 176 [arXiv: 1502.03959] [INSPIRE].

[33] M. Endo, T. Moroi, M.M. Nojiri and Y. Shoji, Renormalization-Scale Uncertainty in the Decay Rate of False Vacuum, JHEP 01 (2016) 031 [arXiv:1511.04860] [INSPIRE]. 